\title{
A Luminous Transient Event in a Sample of WISE-selected Variable AGNs
}

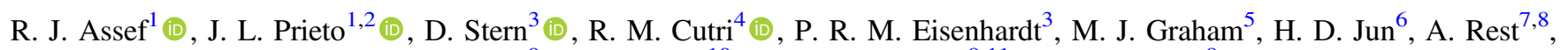 \\ H. A. Flewelling ${ }^{9}$ (D), N. Kaiser ${ }^{10}$ (D), R.-P. Kudritzki ${ }^{9,11}$, and C. Waters ${ }^{9}$ (D) \\ ${ }^{1}$ Núcleo de Astronomía de la Facultad de Ingeniería y Ciencias, Universidad Diego Portales, Av. Ejército Libertador 441, Santiago, Chile; roberto.assef@mail.udp.cl \\ ${ }^{2}$ Millennium Institute of Astrophysics, Santiago, Chile \\ 3 Jet Propulsion Laboratory, California Institute of Technology, 4800 Oak Grove Drive, Pasadena, CA 91109, USA \\ ${ }^{4}$ IPAC, Mail Code 100-22, California Institute of Technology, 1200 E. California Blvd., Pasadena, CA 91125, USA \\ ${ }^{5}$ Cahill Center for Astronomy and Astrophysics, California Institute of Technology, 1216 E. California Blvd., Pasadena, CA 91125, USA \\ ${ }^{6}$ School of Physics, Korea Institute for Advanced Study, 85 Hoegiro, Dongdaemun-gu, Seoul 02455, Republic of Korea \\ ${ }^{7}$ Space Telescope Science Institute, 3700 San Martin Drive, Baltimore, MD 21218, USA
${ }^{8}$ Department of Physics and Astronomy, Johns Hopkins University, 3400 North Charles Street, Baltimore, MD 21218, USA \\ ${ }^{9}$ Institute for Astronomy, University of Hawaii at Manoa, 2680 Woodlawn Drive, Honolulu, HI 96822, USA \\ ${ }^{10}$ Département de Physique, École Normale Supérieure 24 Rue Lhomond F-75231 Paris CEDEX 05, France \\ ${ }^{11}$ University Observatory Munich, Ludwig-Maximilians-University Munich, Scheinerstr. 1, D-81679 Munich, Germany \\ Received 2018 July 19; revised 2018 August 22; accepted 2018 August 28; published 2018 October 5
}

\begin{abstract}
Recently Assef et al. presented two catalogs of active galactic nucleus (AGN) candidates over 30,093 deg $^{2}$ selected $^{2}$ from the Wide-field Infrared Survey Explorer (WISE) observations. From their most reliable sample, Assef et al. identified 45 AGN candidates with the highest variability levels in the AllWISE catalog but that are not blazars. Here we present new spectroscopic observations of some of these targets to further constrain their nature. We also study their optical light curves using observations from the Catalina Real-Time Transient Survey (CRTS) and find that only seven show significant optical variability, and that five of those seven are spectroscopically classified as AGNs. In one of them, WISEA J094806.56+031801.7 (W0948+0318), we identify a transient event in the CRTS light curve. We present a detailed analysis of this transient and characterize it through its CRTS light curve and its multiwavelength spectral energy distribution obtained from GALEX, Pan-STARRS, and WISE observations. We find that the most likely source of the transient is a superluminous supernova (SLSN) in W0948+0318. We estimate the total radiated energy to be $E=(1.6 \pm 0.3) \times 10^{52} \mathrm{erg}$, making it one of the most energetic SLSNe observed. Based on the lack of change in mid-IR color throughout and after the transient event, we speculate that the location of the SLSN is within the torus of the AGN. We identify nine possible analogs to W0948+0318 based on their WISE light curves. None show optically detected transients and hence suggest significant dust obscuration. Finally, we estimate a rate of $>2 \times 10^{-7} \mathrm{yr}^{-1}$ per AGN for these transients under the conservative assumption that none of the identified analogs have a common origin with the transient in W0948+0318.
\end{abstract}

Key words: galaxies: active - infrared: general - quasars: general - supernovae: general

\section{Introduction}

Active galactic nuclei (AGNs) have long been recognized as intrinsically variable sources. This variability has been used not only as a tool to identify AGNs (see, e.g., Padovani et al. 2017, and references therein) but also to study physical characteristics of AGNs themselves, such as their black hole masses (Peterson et al. 2004; Vestergaard \& Peterson 2006) and the structure of their broad-line region (BLR; Denney et al. 2009; Pancoast et al. 2011; Denney 2012). Although the mechanism is yet to be understood, the brightness of their accretion disks changes stochastically as a function of time. This variability has been best studied at optical wavelengths, where it has been shown to follow a well-defined structure function whose amplitude changes as a function of wavelength, black hole mass, and accretion rate (see, e.g., Vanden Berk et al. 2004; MacLeod et al. 2010). For most quasars, the variability can be well characterized by a damped random walk (DRW) process at intermediate and long timescales (Kelly et al. 2009; Kozłowski et al. 2010c; MacLeod et al. 2010; Zu et al. 2011). AGN variability has also been well studied at X-ray wavelengths (e.g., Padovani et al. 2017, and references therein), where emission is dominated by the corona. Studies have shown that variability at these wavelengths qualitatively shares some of the characteristics found in the optical, although with significantly shorter timescales for similar variability amplitudes (e.g., Lawrence et al. 1987) and a break in the power spectral density distribution that depends on black hole mass (e.g., McHardy et al. 2006).

In the mid-IR, however, there have not been many surveys that can address variability. At these wavelengths, the emission is dominated by the so-called dust torus (see, e.g., Nenkova et al. 2008), which simply reprocesses the emission from the accretion disk into the mid-IR. The most notable studies of AGN variability at these wavelengths have come from the Spitzer Space Telescope Deep, Wide-Field Survey (SDWFS; Ashby et al. 2009) and its follow-up, the Decadal IRAC Boötes Survey (DIBS). Based on four and five epochs of Spitzer observations, respectively, Kozłowski et al. (2010a, 2016) found that the variability in the mid-IR is also well characterized by a single structure function with a similar slope to that found at optical wavelengths (Caplar et al. 2017). Additionally, from these observations Kozłowski et al. (2010b) identified a self-obscured luminous supernova $(\mathrm{SN})$ at $z=0.19$ that was only detected in the mid-IR, without a clear optical transient counterpart.

A new window into mid-IR variability has been opened by the Wide-field Infrared Survey Explorer (WISE; Wright et al. 2010), which scanned the entire sky in four mid-IR bands, 
centered at 3.4, 4.6, 12, and $22 \mu \mathrm{m}$ and referred to as $\mathrm{W} 1-\mathrm{W} 4$, respectively. Due to the survey scanning strategy, WISE provided variability on short and long timescales for the entire sky, with the number of observations being a strong function of ecliptic latitude. The WISE all-sky survey was conducted between 2010 January and September, at which point the cryogen was exhausted. The NEOWISE survey (Mainzer et al. 2011) continued the observations in the two shortest wavelength bands, W1 and W2, until 2011 February. After a nearly 2 yr hiatus, the survey then resumed as the NEOWISE Reactivation mission (NEOWISE-R; Mainzer et al. 2014) in 2013 December to continue surveying the sky. The AllWISE data release ${ }^{12}$ made available all the processed observations obtained through 2011, as well as a catalog of all detected sources.

Assef et al. (2018) recently presented a nearly all-sky catalog of AGNs from the WISE mission, selected from their W1 and W2 photometry using a variation of the selection criteria developed by Stern et al. (2012) and Assef et al. (2013). Using the AllWISE data release of the WISE mission, Assef et al. (2018) presented two catalogs: one based on $90 \%$ reliability selection criteria (i.e., where $90 \%$ of the selected objects are expected to be bona fide AGNs), referred to as R90, with approximately 4.5 million AGN candidates, and one based on $75 \%$ completeness criteria (i.e., where $75 \%$ of AGNs detected by WISE are recovered), referred to as C75, with approximately 21 million AGN candidates (see Assef et al. 2018, for details). Due to its survey design, WISE naturally obtains variability information on two timescales: one of approximately $3 \mathrm{hr}$, equal to twice the orbital period of the spacecraft, and another one regulated by the orbital motion of Earth, which corresponds to $0.5 \mathrm{yr}$ in the ecliptic and becomes significantly smaller near the ecliptic poles, achieving visibility during the full survey duration at the latter. For the rest of this article, we refer to each group of observations separated by the $\sim 0.5 \mathrm{yr}$ timescale as the epochs of the WISE observations.

Assef et al. (2018) noted that 687 of the $\sim 4.5$ million AGN candidates in the R90 catalog $(0.015 \%)$ were classified as highly variable (i.e., variability flag var_flg $=9$ ) in the AllWISE database in both W1 and W2. In brief, AllWISE characterizes the probability that the flux of any given source in a specific band is not constant with time in individual exposures, based on the flux uncertainties and the temporal correlation with the flux at different bands. A variability flag of var_flg $=9(0)$ indicates the highest (lowest) probability of variability. Sources with var_flg of 8 or 9 are most likely truly variable in a band, while sources with var_flg $\leqslant 5$ are most likely not real variables. As expected, Assef et al. (2018) found the majority of these sources to be likely blazars, as of the 207 sources located within the FIRST survey (Becker et al. 1995) footprint, $162(78 \%)$ have well-detected radio emission in the FIRST catalog ${ }^{13}$ within $5^{\prime \prime}$ from the WISE position. ${ }^{14}$ For the other 45 sources covered by FIRST, however, the underlying mechanism for their variability remains unclear. One of these sources, WISEA J142846.71+172353.1, was identified as a

\footnotetext{
12 http://wise2.ipac.caltech.edu/docs/release/allwise/expsup/

13 http://sundog.stsci.edu/cgi-bin/searchfirst

14 One additional source, WISEA J151215.73+020316.9, is associated with two radio sources, $9^{\prime \prime}$ and $13^{\prime \prime}$ away, and has been associated with the gammaray source 3FGL J1512.2+0202, making it most likely a blazar. For compatibility with the list of targets of Assef et al. (2018), we keep it in the list of radio-undetected sources discussed in the next sections.
}

changing-look AGN by Assef et al. (2018) by comparing spectroscopy from the Sloan Digital Sky Survey (SDSS) DR13 (Albareti et al. 2017) obtained in 2008 ( 2 yr before the WISE survey observations) with dedicated follow-up spectroscopic observations obtained in 2017. Due to the large change in flux observed in the WISE bands, Assef et al. (2018) were able to determine the most likely source of the dramatic AGN variability to be a drop in the accretion rate rather than a change in obscuration (see Ross et al. 2018; Stern et al. 2018, for detailed discussion of the physics behind similar objects).

In this article we focus on these 45 highly variable mid-IR AGNs identified by Assef et al. (2018) that are within the FIRST survey footprint but are undetected at radio wavelengths. In Section 2 we discuss the spectroscopic classification of these sources, presenting new observations to update the discussion of Assef et al. (2018). In this section we also study the optical light curves of these sources and identify one source, WISEA J094806.56+031801.7 (W0948+0318), that shows a unique light curve. In Section 3 we present additional observations of W0948+0318, and in Section 4 we present a detailed discussion of its light curve. In Section 5 we present a discussion of the possible sources for the observed transient, and in Section 6 we present some possible analogs to this transient that have very similar mid-IR light curves but show no significant optical variability. Throughout the paper, magnitudes are presented in their natural system unless stated otherwise, namely, Vega for $V_{\mathrm{CSS}}$ and the WISE bands, and $\mathrm{AB}$ for gri and the GALEX bands. We assume a flat $\Lambda$ CDM cosmology with $\Omega_{\mathrm{M}}=0.286$ and $H_{0}=69.3 \mathrm{~km} \mathrm{~s}^{-1} \mathrm{Mpc}^{-1}$.

\section{Highly Variable Radio-undetected WISE AGNS}

In this section we focus on the 45 highly variable AGN candidates identified by Assef et al. (2018) from their 90\% reliability WISE AGN catalog (R90) that are within the FIRST survey footprint but are undetected at radio wavelengths.

\subsection{Spectroscopic Classification}

Assef et al. (2018) studied the spectroscopic classification of 34 of these 45 sources, with 32 of them taken from either SDSS or SIMBAD, ${ }^{15}$ and two of them determined from recent observations carried out using the DBSP instrument at the Palomar Observatory 200-inch telescope (see Assef et al. 2018, for details). They found that 31 of the 34 objects were consistent with an AGN classification based on their optical spectra, and that the remaining three objects were stellar contaminants (consistent with the expected $90 \%$ reliability of the sample). Two of the Galactic interlopers were classified as carbon stars. The AGNs have redshifts between 0.0365 and 0.5550 and have a diversity of spectral features.

Of these 31 objects consistent with an AGN spectroscopic classification, 18 were classified as QSOs, one as a Seyfert 1 galaxy, one as a red type $1 \mathrm{AGN}$, and one as a type 2 AGN. Six other objects were classified as "Galaxy AGN" by SDSS, and inspection of their spectra indicates either that they have broad bases to their $\mathrm{H} \alpha$ emission lines but only show narrow, if any, $\mathrm{H} \beta$ emission or that they have line ratios indicative of obscured AGN activity. The remaining four objects were classified as galaxies, but Assef et al. (2018) find, upon inspection of their spectra, that all show hints of broad bases to their $\mathrm{H} \alpha$ emission

\footnotetext{
${ }^{15}$ http://simbad.u-strasbg.fr/simbad/
} 


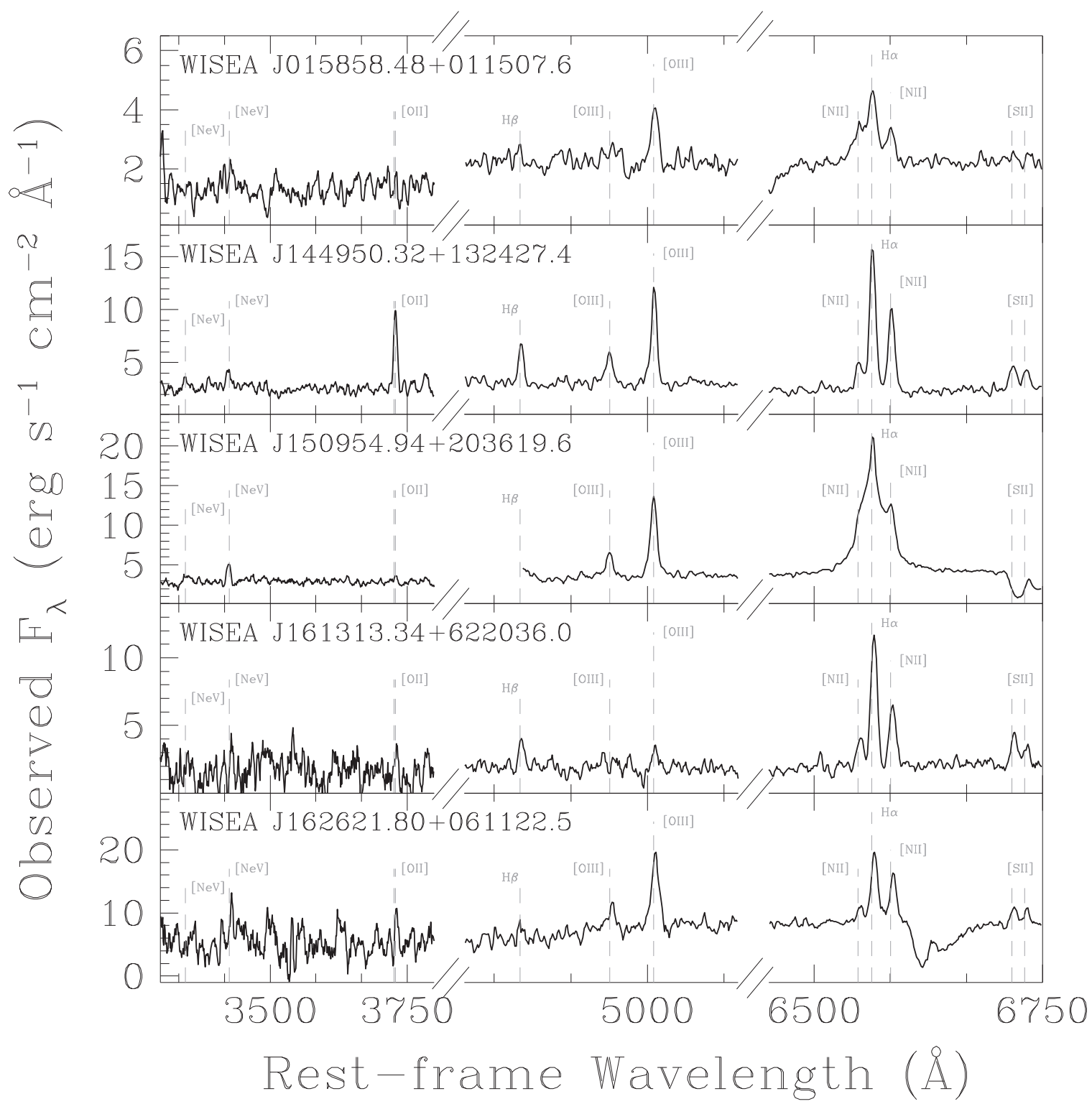

Figure 1. Optical spectra of five radio-undetected, highly variable AGN candidates. All spectra were obtained with the DBSP instrument at the Palomar Observatory 200-inch telescope. The gap in the spectrum of WISEA J150954.94+203619.6 is due to the dichroic used in the observations. Note that the $x$-axis is split into three different wavelength ranges for clarity.

lines, suggesting an important AGN contribution. Hence, of the 31 highly variable AGNs with spectroscopic classifications, 19 $(61 \%)$ are unobscured AGNs, and the remaining $12(39 \%)$ show some degree of likely obscuration by being classified as either a red type 1, intermediate-type, or type 2 AGN.

To provide a more complete spectroscopic analysis, we have obtained spectroscopic observations of an additional four targets from the sample of 45 . We also obtained spectroscopic observations of WISEA J150954.94+203619.6, for which, although discussed by Assef et al. (2018), only a photometric classification of "Possible AGN" and a photometric redshift of 0.41492 were reported by SIMBAD from the work of Oyaizu et al. (2008) and Szabo et al. (2011). We obtained the spectra using the DBSP optical spectrograph at the Palomar Observatory 200-inch telescope, on the nights of UT 2016 February 6, UT 2017 April 22, UT 2017 August 6, and UT 2018 January 6. We used the same instrumental setup as described in Assef et al. (2018), namely, a slit with a 1". 5 width, the D55 dichroic, the 600 line $\mathrm{mm}^{-1}$ grating ( $4000 \AA$ blaze) on the blue arm, and the 316 line $\mathrm{mm}^{-1}$ grating (7500 $\AA$ blaze) on the red arm. Reductions were carried out in a standard manner using IRAF. ${ }^{16}$

The spectra of the five targets are shown in Figure 1. Two of the sources, WISEA J015858.48+011507.6 $(z=0.184)$ and WISEA J150954.94+203619.6 $(z=0.131)$, have broad $\mathrm{H} \alpha$ emission lines, consistent with an AGN classification. The latter has a spectroscopic redshift well below the photometric redshift of 0.41492 . Two other sources, WISEA J144950.32+132427.4 $(z=0.198)$ and WISEA J162621.80+061122.5 $(z=0.149)$, have high [O III]-to- $\mathrm{H} \beta$ ratios and detections of [ $\mathrm{Ne} \mathrm{V}]$ emission, consistent with an obscured AGN classification. The other source, WISE J161313.34+622036.0 $(z=0.262)$, has only narrow emission lines that do not suggest an AGN classification. This implies that the source is likely a contaminant to the R90

${ }^{16}$ http://iraf.noao.edu 
Table 1

Properties of Radio-quiet, Highly Variable WISE AGNs

\begin{tabular}{|c|c|c|c|c|}
\hline $\begin{array}{l}\text { WISE ID } \\
\text { (WISEA) }\end{array}$ & Redshift & CRTS $\chi_{\nu}^{2}$ & Classification & Ref \\
\hline $\mathrm{J} 000011.72+052317.4$ & 0.0400 & 2.4 & Seyfert 1 & SIMBAD \\
\hline J014004.69-094230.4 & 0.1461 & 2.3 & QSO & SDSS \\
\hline J015858.48+011507.6 & 0.184 & 2.3 & AGN & P200/DBSP \\
\hline J090546.35+202438.2 & $\ldots$ & 20.5 & Carbon Star & SIMBAD \\
\hline J091225.00+061014.8 & 0.1453 & 0.6 & Galaxy $^{\mathrm{a}}$ & SDSS \\
\hline J094806.56+031801.7 & 0.2073 & 4.9 & QSO & SDSS \\
\hline J095824.97+103402.4 & 0.0417 & 1.3 & Galaxy AGN & SDSS \\
\hline $\mathrm{J} 100933.13+232255.7$ & 0.0719 & 0.4 & Galaxy AGN & SDSS \\
\hline $\mathrm{J} 101536.17+221048.9$ & $\ldots$ & 2.2 & $\ldots$ & $\ldots$ \\
\hline $\mathrm{J} 104241.08+520012.8$ & 0.1365 & 2.1 & QSO & SDSS \\
\hline $\mathrm{J} 112537.83+212042.2$ & 0.0894 & 2.0 & QSO & SDSS \\
\hline J130155.84+083631.7 & $\ldots$ & 16.4 & Carbon Star & SIMBAD \\
\hline $\mathrm{J} 130716.98+450645.3$ & 0.0843 & 0.9 & QSO & SDSS \\
\hline J130819.11+434525.6 & 0.0365 & 0.4 & Galaxy AGN & SDSS \\
\hline $\mathrm{J} 140033.66+154432.1$ & 0.2152 & 2.2 & QSO & SDSS \\
\hline $\mathrm{J} 141053.43+091027.0$ & 0.1781 & 7.1 & QSO & SDSS \\
\hline $\mathrm{J} 141105.45+294211.8$ & 0.0724 & 0.5 & QSO & SDSS \\
\hline J141758.60+091609.7 & 0.1389 & 3.6 & QSO & SDSS \\
\hline $\mathrm{J} 142747.45+165206.0$ & 0.1435 & 0.7 & QSO & SDSS \\
\hline $\mathrm{J} 142846.71+172353.1$ & 0.1040 & 0.7 & QSO & SDSS \\
\hline J143457.66-051038.9 & $\ldots$ & 0.3 & $\ldots$ & $\ldots$ \\
\hline $\mathrm{J} 144039.30+612748.1$ & 0.0811 & $\ldots$ & QSO & SDSS \\
\hline $\mathrm{J} 144131.81+321612.9$ & 0.1993 & 0.7 & QSO & SDSS \\
\hline $\mathrm{J} 144439.59+351304.7$ & 0.0790 & 0.4 & Galaxy $^{\mathrm{a}}$ & SDSS \\
\hline $\mathrm{J} 144510.14+304957.1$ & 0.2754 & 6.1 & QSO & SDSS \\
\hline J144603.98-013203.4 & 0.0840 & 0.3 & Galaxy AGN & SDSS \\
\hline $\mathrm{J} 144950.32+132427.4$ & 0.198 & 0.9 & AGN & P200/DBSP \\
\hline $\mathrm{J} 145222.03+255152.0$ & 0.1204 & 0.4 & QSO & SDSS \\
\hline $\mathrm{J} 150842.68+212132.1$ & $\ldots$ & 1.2 & $\ldots$ & $\ldots$ \\
\hline J150954.94+203619.6 & 0.131 & 0.6 & AGN & P200/DBSP \\
\hline $\mathrm{J} 151215.73+020316.9$ & 0.2199 & 9.7 & Galaxy $\mathrm{AGN}^{\mathrm{b}}$ & SDSS \\
\hline $\mathrm{J} 151444.52+364237.9$ & 0.1944 & 1.0 & QSO & SDSS \\
\hline J151518.56+312937.5 & 0.1036 & 0.5 & QSO & SDSS \\
\hline $\mathrm{J} 154324.98+545652.0$ & $\ldots$ & 1.2 & $\ldots$ & $\ldots$ \\
\hline $\mathrm{J} 155223.29+323455.0$ & 0.1277 & 1.5 & Galaxy $^{\mathrm{a}}$ & SDSS \\
\hline $\mathrm{J} 161313.34+622036.0$ & 0.262 & 1.4 & Galaxy & P200/DBSP \\
\hline J161846.36+510035.1 & 0.3189 & 1.9 & QSO & SDSS \\
\hline $\mathrm{J} 162140.25+390105.1$ & 0.0642 & 0.6 & Galaxy AGN & SDSS \\
\hline $\mathrm{J} 162621.80+061122.5$ & 0.149 & 1.0 & AGN & P200/DBSP \\
\hline J163214.82+634854.5 & $\ldots$ & 1.5 & $\ldots$ & $\ldots$ \\
\hline J163518.38+580854.6 & $\ldots$ & 1.2 & Star & SIMBAD \\
\hline J165336.08+594347.1 & $\cdots$ & 0.9 & $\ldots$ & $\ldots$ \\
\hline J165932.68+470448.5 & $\ldots$ & 0.8 & $\ldots$ & $\ldots$ \\
\hline J170741.24+523714.4 & $\ldots$ & 0.8 & $\ldots$ & $\ldots$ \\
\hline $\mathrm{J} 213604.22-050152.0$ & 0.1284 & 0.8 & Galaxy $^{\mathrm{a}}$ & SIMBAD \\
\hline
\end{tabular}

Notes.

${ }^{\text {a }}$ Although the object is classified as a galaxy in SDSS or SIMBAD, the H $\alpha$ emission line shows a broad base, suggesting the presence of an AGN.

${ }^{\mathrm{b}}$ Most likely a blazar owing to its association with the gamma-ray source 3FGL J1512.2+0202 and large-scale radio emission.

sample, as a certain number of low-redshift, strongly star-forming galaxies are expected to contaminate the sample (see Assef et al. 2018, for details). An updated version of Table 4 of Assef et al. (2018) is provided in Table 1, including the spectroscopic redshifts and classifications of these five targets, as well as all the other highly variable AGNs with and without spectroscopic information.

\subsection{Light Curves}

To further characterize these 45 highly variable WISE AGN candidates, we cross-matched our sample with optical photometry available from the Catalina Real-Time Transient Survey (CRTS; Drake et al. 2009) DR2 data release. ${ }^{17}$ CRTS DR2 provides optical light curves in the $V_{\mathrm{CSS}}$ band for objects with declinations in the range $-75^{\circ} \lesssim \delta \lesssim 65^{\circ}$ and farther than $10^{\circ}-15^{\circ}$ from the Galactic plane with $V_{\mathrm{CSS}}$-band magnitudes between 11.5 and 21.5. We obtained CRTS optical light curves for 44 of the 45 highly variable, radio-undetected WISE AGN candidates. The source without a CRTS counterpart, WISEA $\mathrm{J} 144039.30+612748.1$, is outside the footprint of the CRTS survey.

\footnotetext{
$\overline{{ }^{17} \text { http://nesssi.cacr.caltech.edu/DataRelease/ }}$
} 


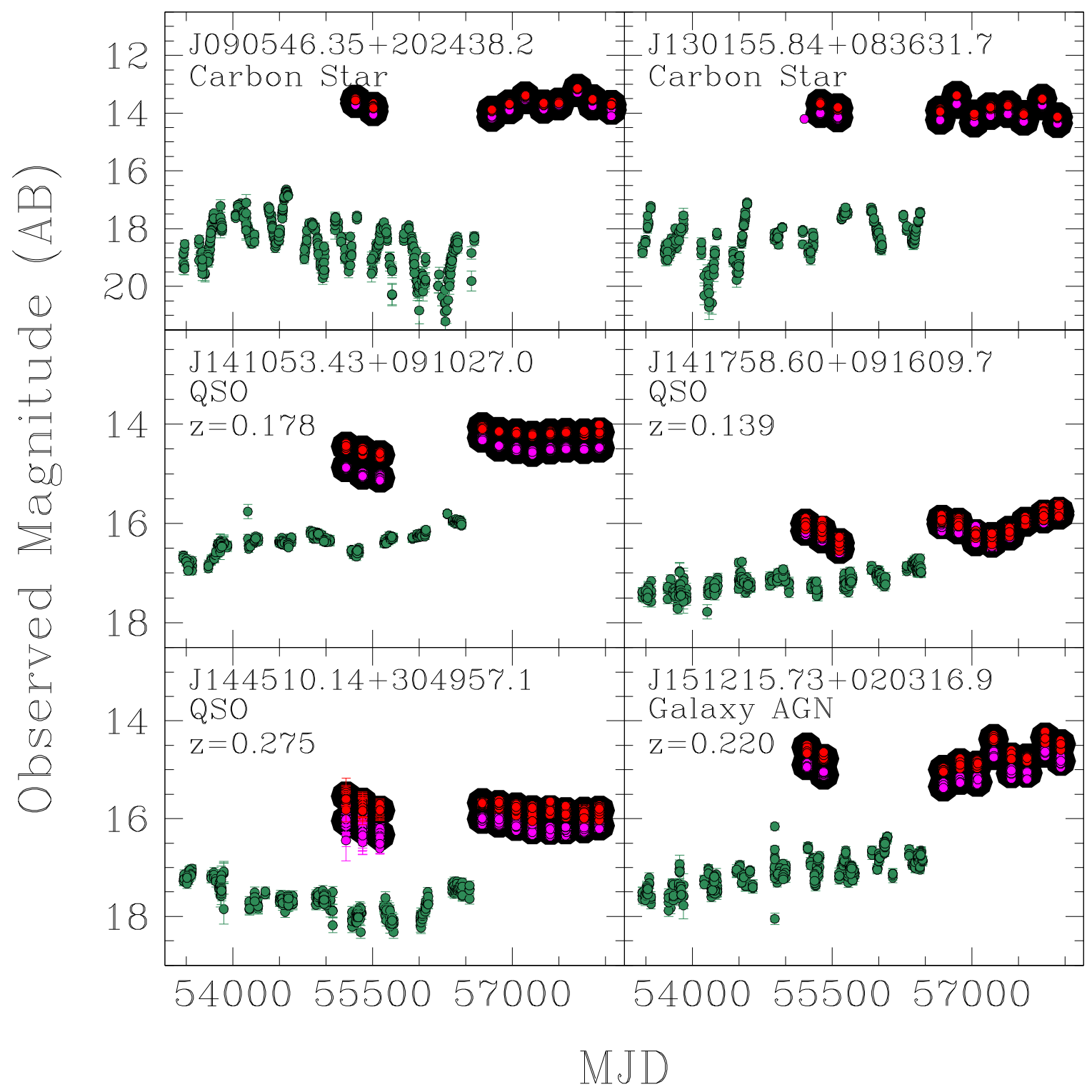

Figure 2. Light curves of six of the seven radio-undetected, highly mid-IR variable AGN candidates with high optical variability (see text for details). The other target is shown in Figure 3. The figure shows the optical CRTS $V_{\text {CSS }}$ band (green) and WISE W1 (magenta) and W2 (red) bands. For W1 and W2, the small circles show the individual frame photometry in the AllWISE and NEOWISE-R surveys. The large black circles show the median of each epoch. To minimize the range of magnitudes between bands, all magnitudes are shown in the AB system. To convert W1 (W2) from Vega to AB, we add $2.68 \mathrm{mag}$ (3.32 mag).

Inspection of the optical light curves reveals that while some objects show strong optical variability, the majority of objects are relatively quiescent, despite being highly variable in the mid-IR. We quantify the level of variability of sources by comparing how much the light curve deviates from a constant flux. Specifically, we fit a constant flux to the $V_{\mathrm{CSS}}$ light curve and measure $\chi_{\nu}^{2}$, the $\chi^{2}$ per degree of freedom, of the fit. We eliminate the $5 \%$ most discrepant points from each light curve to ensure that high $\chi_{\nu}^{2}$ values are driven by real variability and not outliers. These values are shown for all 44 light curves in Table 1. Note that $22(50 \%)$ have $\chi_{\nu}^{2}<1$ and $30(67 \%)$ have $\chi_{\nu}^{2}<2$, confirming that the majority of targets have optical light curves that are not strongly variable. There are, however, seven targets that have $\chi_{\nu}^{2}>3$, implying stronger optical variability. Figures 2 and 3 show the optical and mid-IR light curves of these seven targets. The light curves for the rest of the sample are presented in Section 6 and Appendix B. The CRTS light curves have been corrected for foreground reddening using $V$-band extinction determined from the extinction maps of Schlafly \& Finkbeiner (2011) queried through the NASA/ IPAC Extragalactic Database (NED) Coordinate \& Extinction Calculator. ${ }^{18}$ To extend their mid-IR light curves, we have also included the observations of the NEOWISE-R mission (Mainzer et al. 2014) from the 2018 Data Release. ${ }^{19}$

W0948+0318 is unlike any of the other sources presented above, with very strong epoch-to-epoch variability during the AllWISE observations, but shows little to no variability during the NEOWISE-R observations. Concurrent to the AllWISE observations, CRTS shows a very strong optical transient event whose light-curve decay qualitatively matches the behavior observed in the WISE bands. While other highly variable radio-

\footnotetext{
18 https://ned.ipac.caltech.edu/forms/calculator.html

19 http://wise2.ipac.caltech.edu/docs/release/neowise/
} 


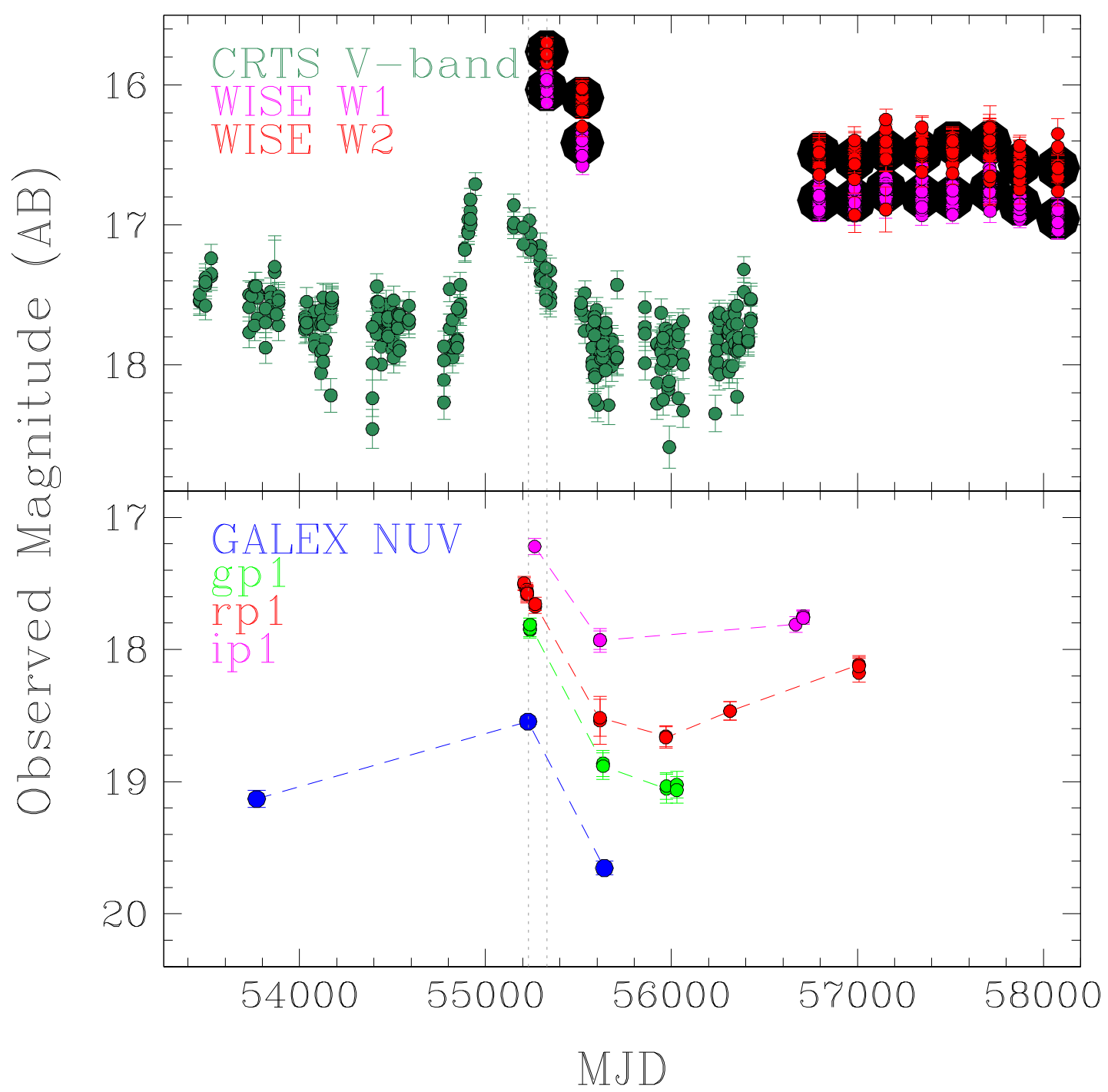

Figure 3. Light curve of WISE J094806.56+031801.8 (W0948+0318). Symbols in the top panel have the same definition as in Figure 2. The bottom panel shows the GALEX NUV band (blue) and Pan-STARRS $g$ (green), $r$ (red), and $i$ (magenta). All magnitudes are in the AB system. The vertical gray dotted lines show the dates at which we model the SED of the transient in Section 4.3.

undetected AGN candidates have similar WISE light curves (see Section 6 for details), none show significant optical variability, and of all targets with high optical variability, W0948+0318 is the only one whose optical light curve is dominated by a single transient event.

Recently, Graham et al. (2017) studied AGN light curves in the CRTS data and independently identified W0948+0318 as one of 51 objects whose light curves strongly deviated from a DRW model. They concluded that AGN flaring events are incompatible with the stochastic variability properties of AGNs, and instead proposed a number of different mechanisms for these optical transient events. Specifically, they discussed these sources in the context of microlensing events by stars in the Milky Way, superluminous SNe (SLSNe), tidal disruption events (TDEs), and black hole binary mergers. While the data available did not allow us to differentiate between most of these scenarios, they were able to put constraints on the possible microlensing nature of these sources, and for the specific case of W0948+0318, Graham et al. (2017) find that the microlensing explanation is unlikely given the asymmetry of the optical light curve (see Figure 3). Here, we can further rule out this scenario since the transient event is also present in the
WISE bands. While the accretion disk can be of comparable size to the Einstein ring of a stellar lens, the observed emission in the $\mathrm{W} 1$ and $\mathrm{W} 2$ bands is dominated by dust in the torus, which is at significantly larger physical scales.

In the following sections we use additional observations to constrain the nature of the transient event in W0948+0318.

\section{Additional Observations of W0948+0318}

\subsection{GALEX Photometry}

The Galaxy Evolution Explorer (GALEX; Martin et al. 2005), through its DR7 data release, provides three epochs of NUV photometry for W0948+0318, as well as one epoch of photometry in the FUV band. The earliest epoch provided comes from the co-addition of two exposures obtained approximately 1 month apart from each other, on UT 2006 February 03 and 2006 March 16, well before the optical transient. The combined exposure time is $217 \mathrm{~s}$ in each band. For simplicity we assign the epoch of this observation to the date of the first exposure. This does not impact any of the results presented in this article. The second epoch provided also comes from the co-addition of two exposures, but this time 
taken only a few hours apart from each other on UT 2010 February 02. This observation was obtained only in the NUV band, with a total exposure time of 2119 s. The third and final epoch comes from a single NUV exposure of $1648.15 \mathrm{~s}$ obtained on UT 2012 March 06.

The target is detected in the NUV band in all three visits, with reported respective $\mathrm{AB}$ magnitudes of $19.410 \pm 0.065$, $18.825 \pm 0.021$, and $19.933 \pm 0.052$. The target was detected in the only FUV observation obtained, with a magnitude of $19.472 \pm 0.105$. The NUV light curve is presented in the bottom panel of Figure 3. By chance, the second epoch of GALEX observations was obtained shortly after the maximum of the transient event, and the third epoch was obtained shortly after the transient had faded away. The first epoch, instead, was obtained significantly before the transient event, toward the beginning of the CRTS observations. For the spectral energy distribution (SED) analysis presented in Section 4, we correct the GALEX photometry for foreground reddening using the coefficients of Yuan et al. (2013) to extrapolate the $u$-band reddening reported by NED from the Schlafly \& Finkbeiner (2011) extinction maps to the NUV band. For FUV we assume the same extinction as for NUV owing to the uncertainties in the coefficient reported by Yuan et al. (2013), and we add to the uncertainty in FUV a factor based on the ranges of coefficients they considered.

\subsection{Pan-STARRS Photometry}

Starting in 2010, the Panoramic Survey Telescope and Rapid Response System (Pan-STARRS; Chambers et al. 2016; Flewelling et al. 2016) has used a $1.8 \mathrm{~m}$ telescope to repeatedly image the northern sky in the grizy bands. During its first years of operations, the survey observed W0948+0318 in the $g, r$, and $i$ bands during the transient event, as well as after it had disappeared. Individual observations were obtained with exposure times of either 52 or $43 \mathrm{~s}$ in the $g$ band, with exposure times ranging between 30 and $60 \mathrm{~s}$ in the $r$ band, and with exposure times of either 32 or $45 \mathrm{~s}$ in the $i$ band. The basic data processing of these individual PS1 images is performed by the PS1 IPP system (Magnier et al. 2016; Waters et al. 2016). Downstream processing is then performed with the photpipe pipeline, which has been used for various time-domain surveys like SuperMACHO, ESSENCE, and the PS1 Medium Deep Field survey (Rest et al. 2005, 2014). Images in the $z$ and $y$ bands were only obtained after the transient had faded away. We measured the flux of the source for every epoch of Pan-STARRS using $4^{\prime \prime}$ diameter apertures. We obtained the zero-point of the photometry by comparing the flux of all stars in the field against their magnitudes reported by the Pan-STARRS DR1 for the stacked frames (photometry for individual exposures is not available in DR1). We correct the photometry for foreground reddening using the extinction maps of Schlafly \& Finkbeiner (2011) queried through NED. The respective light curves are shown in the bottom panel of Figure 3.

In the co-added frames released as part of the Pan-STARRS DR1, W0948+0318 is marked as extended. However, in the $g$, $r$, and $i$ bands the difference between the profile fitting magnitude and the Kron-radius magnitude is $\sim 0.05 \mathrm{mag}$, indicating that the majority of the flux is consistent with a point source and hence that the source is only marginally resolved in these bands.

\subsection{SDSS Photometry and Spectroscopy}

The SDSS DR13 reports photometry for W0948+0318 obtained before the transient event on MJD 51,960, approximately 2800 days $(\sim 7.5 \mathrm{yr})$ before the transient event. For the analysis of the pre-transient SED presented in the next section, we specifically use the ModelMag $\mathrm{AB}$ magnitudes of $u=$ $18.80 \pm 0.02, \quad g=18.50 \pm 0.01, \quad r=18.21 \pm 0.01, \quad i=$ $17.87 \pm 0.01$, and $z=17.94 \pm 0.02$.

W0948+0318 is spectroscopically identified as a type 1 AGN at a redshift of $z=0.20728 \pm 0.00003$ in the SDSS DR13. The SDSS optical spectrum is shown in Figure 4 and was obtained in MJD 52,266, approximately 2500 days ( $\sim 7 \mathrm{yr}$ ) before the event. The hydrogen emission lines are clearly broadened with respect to the rest of the emission lines, consistent with the type 1 AGN classification. The continuum luminosity at $5100 \AA$ is measured to be $L_{5100}=8.5 \pm 0.5 \times 10^{43} \mathrm{erg} \mathrm{s}^{-1}$. From the photometric SED modeling of W0948+0318 before the transient event that will be presented in Section 4.1 , we estimate that $41 \% \pm$ $6 \%$ of the emission observed at $5100 \AA$ comes from the AGN, with the rest coming from the host galaxy. Hence, the AGN continuum luminosity at $5100 \AA$ is $L_{5100}^{\mathrm{AGN}}=3.5 \pm 0.5 \times 10^{43} \mathrm{erg} \mathrm{s}^{-1}$.

The SDSS DR13 reports line widths for both the $\mathrm{H} \alpha$ and $\mathrm{H} \beta$ emission lines of $\sigma=886.5 \pm 7.6 \mathrm{~km} \mathrm{~s}^{-1}$, while all narrow emission lines, including [N II] and [O III], have reported line widths of $\sigma=132.8 \pm 2.0 \mathrm{~km} \mathrm{~s}^{-1}$. Using the AGN continuum luminosity at $5100 \AA, L_{5100}^{\mathrm{AGN}}$, and the line dispersion of $\mathrm{H} \beta$, we infer a black hole mass of $M_{\mathrm{BH}}=1.2 \pm 0.4 \times 10^{7} M_{\odot}$ using the radius-luminosity relation of Bentz et al. (2009) and the $f$ factor of Collin et al. (2006), following Equation (4) of Assef et al. (2011). Using the bolometric correction for $L_{5100}^{\mathrm{AGN}}$ of Gallagher et al. (2007), we infer a bolometric luminosity of $L_{\mathrm{Bol}}=3.6 \pm 1.6 \times 10^{44} \mathrm{erg} \mathrm{s}^{-1}$, which implies an Eddington ratio of $L_{\mathrm{Bol}} / L_{\mathrm{Edd}}=0.25 \pm 0.14$. Note, however, that these could be considered as upper limits, as the bolometric correction used includes the dust emission, which is just reprocessed light from the accretion disk. We note that, assuming a Gaussian profile for the $\mathrm{H} \alpha$ and $\mathrm{H} \beta$ emission lines, their corresponding FWHM would be $2088 \pm 18 \mathrm{~km} \mathrm{~s}^{-1}$, just above the usual threshold of FWHM $<2000 \mathrm{~km} \mathrm{~s}^{-1}$ used to classify narrow-line Seyfert 1s (Osterbrock \& Pogge 1985; Goodrich 1989). If W0948+0318 is indeed a narrow-line Seyfert 1, its Eddington ratio would be toward the lower end of the range observed by, e.g., Grupe (2004), which goes from $L_{\mathrm{Bol}} / L_{\mathrm{Edd}} \sim 0.1$ to somewhat above the Eddington limit.

\section{Analysis of the Event}

\subsection{Pre-transient SED of W0948+0318}

We start by modeling the pre-transient SED of W0948 +0318 , constructed from the first epoch of GALEX FUV and NUV observations (see Section 3.1), the SDSS photometry reported in Section 3.3, and the Two Micron All Sky Survey (2MASS; Skrutskie et al. 2006) photometry in the $J, H$, and $K_{s}$ bands reported in the 2MASS All-Sky Point Source Catalog queried through the Infrared Science Archive (IRSA). The 2MASS observations were carried out in MJD 51,571.31, approximately $1.5 \mathrm{yr}$ before the SDSS observations and approximately $9 \mathrm{yr}$ before the transient event.

We model these bands using the SED templates and algorithm of Assef et al. (2010). In short, we model the SED of the object through a non-negative linear combination of an 


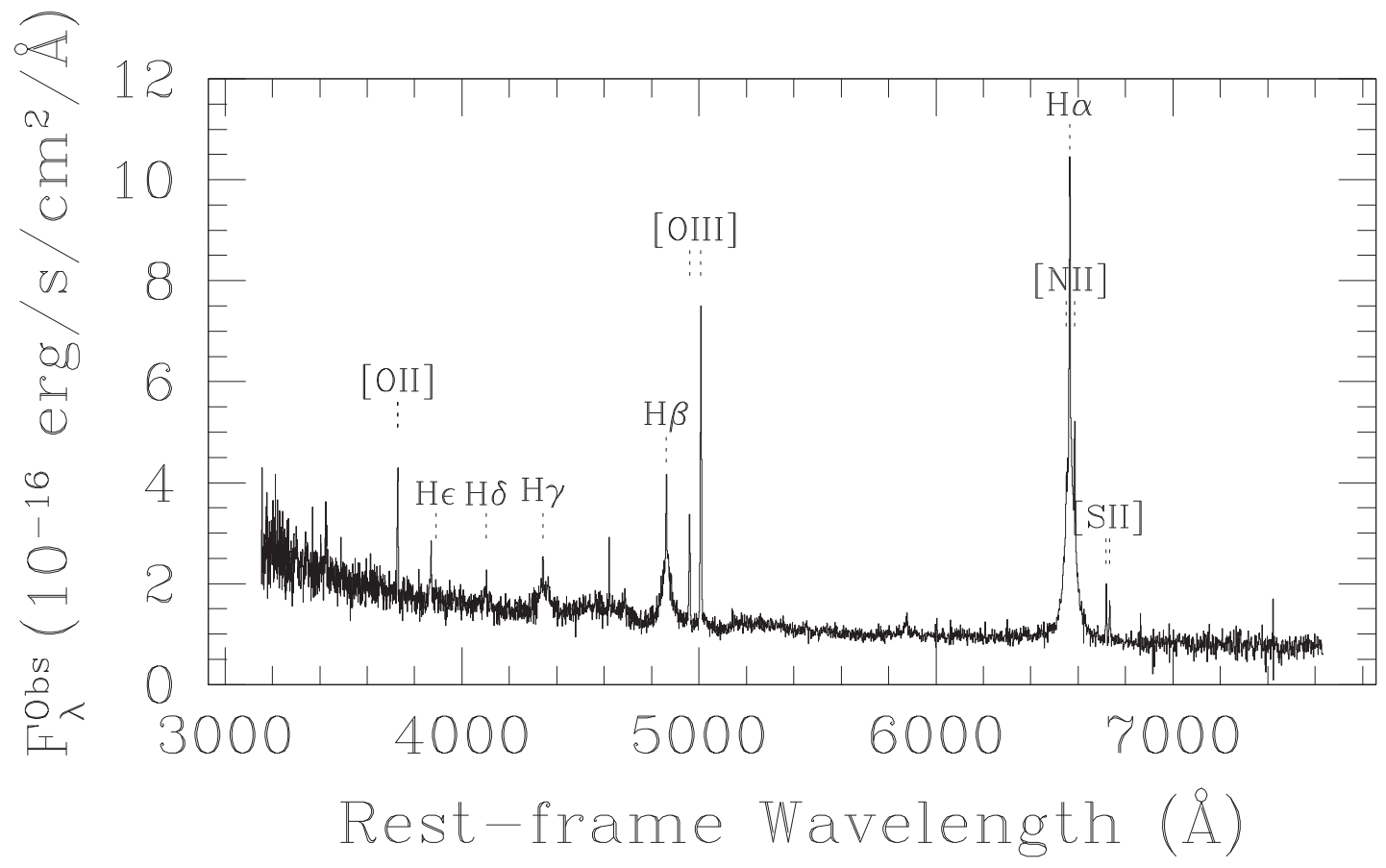

Figure 4. SDSS spectrum of W0948+0318.

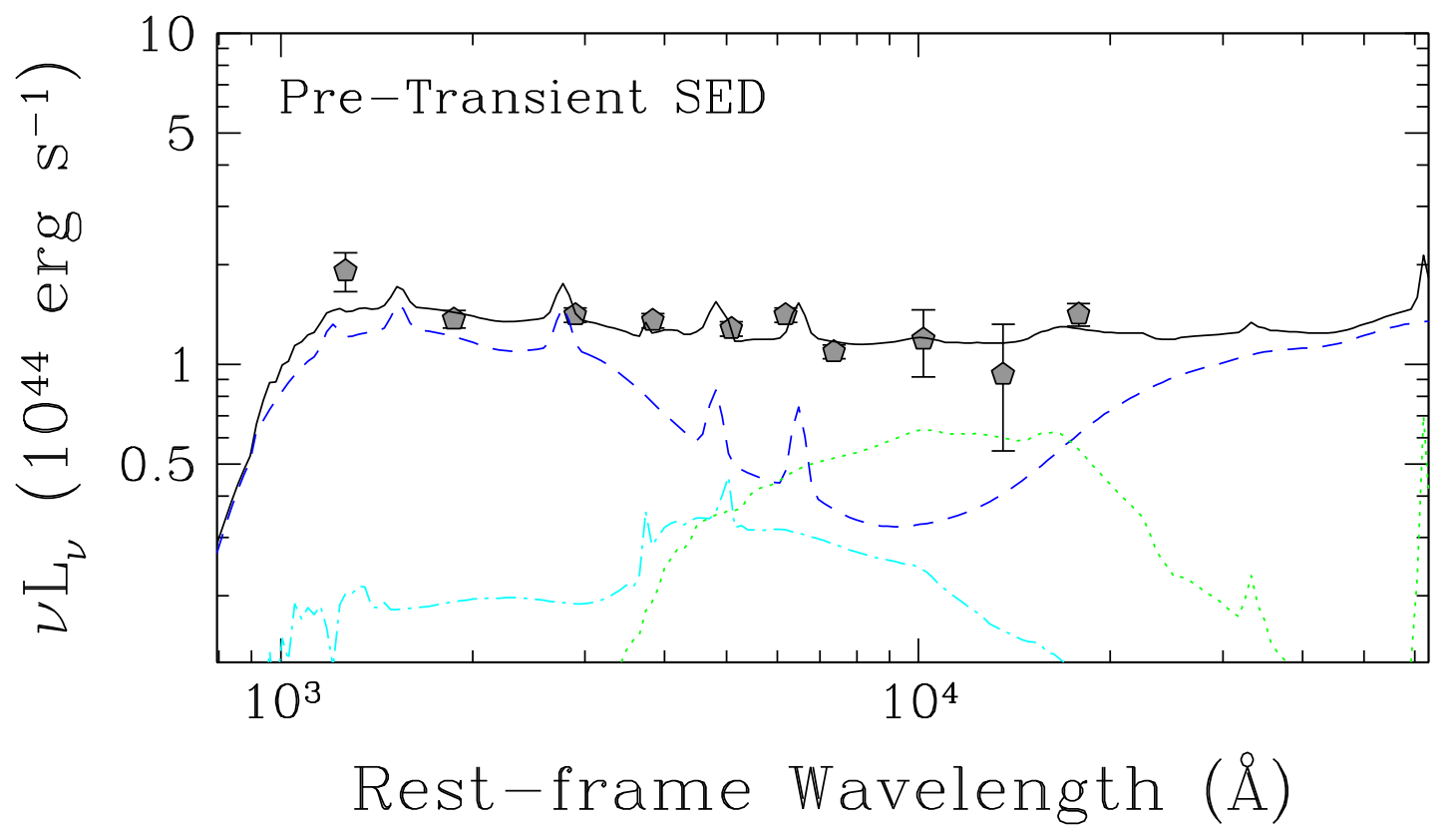

Figure 5. SED of W0948+0318 before the transient occurred. From shortest to longest wavelength, the gray pentagons show the monochromatic luminosity of W0948 +0318 in the GALEX FUV and NUV bands; the SDSS $u, g, r, i$, and $z$ bands; and the 2MASS $J, H$, and $K_{s}$ bands. The solid black line shows the best-fit SED model, composed of a mildly reddened AGN (dashed blue line), the Sbc galaxy (dotted green line), and the Im galaxy (dot-dashed cyan line) templates of Assef et al. (2010).

empirical AGN template and three empirical galaxy SED templates, roughly corresponding to a late-type galaxy (E template), an intermediate spiral (Sbc template), and a local starburst (Im template). We also fit for the obscuration of the AGN template (see Assef et al. 2010, for details). We note that no effort was taken to correct for the intrinsic variability of the AGN between the different observations. Additionally, we are considering multiwavelength photometry obtained through a number of different methods that likely drive systematic differences between the bands, so we enforce a minimum flux error of $5 \%$ for each band.
The best-fit SED model is shown in Figure 5 and has $\chi_{\nu}^{2}=2.6$. As expected, the source is dominated by an AGN component in the UV and optical, while the host galaxy emission dominates the near-IR. The AGN contributes $76 \%$ of the luminosity in the wavelength range between 0.1 and $30 \mu \mathrm{m}$. The best-fit SED model requires a mild reddening of $E(B-V)=0.07$ over the AGN component, although we note that the FUV band is significantly above the best-fit SED. This suggests that the true $E(B-V)$ may be closer to zero, but that the reddening is being used to better accommodate the $u$-NUV color, which could be affected by the systematic issues 
described above. Furthermore, FUV likely also has a significant contribution from Ly $\alpha$, which could be stronger than that of the SED template from Assef et al. (2010).

\subsection{Optical Light Curve}

Figure 3 shows the light curve of the event. The optical light curve shows a single strong outburst event, starting at about MJD 54,800, with lower amplitude variability over the entire time span of the CRTS observations. The transient event is clearly asymmetric in the optical, brightening in $\sim 200$ days, and decaying over the next $\sim 600$ days. The peak of the light curve is unfortunately not well mapped by the CRTS observations, so we assume it to be at the maximum flux detected, on MJD 54,944.23.

Figure 3 also shows the AllWISE light curve, to which we have added the W1 and W2 observations of the latest data release of the NEOWISE-R mission. The small red and magenta data points in Figure 3 show the individual measurements of each visit, while the large data points show the median photometry of those visits grouped by their halfyear epoch. The error bars of each individual visit are somewhat underestimated, so we do not study mid-IR variability on the shortest timescales. The event was observed by the WISE survey while fading after its peak magnitude, and it shows no significant variability in the $\sim 6$-month timescales during the first six epochs of the NEOWISE-R mission, while fading in the last two epochs. The W1-W2 color of W0948 +0318 shows almost no change between the AllWISE and NEOWISE-R epochs, being consistent with the AGN classification of Assef et al. (2018) at all times. The W1-W2 color in the AllWISE source catalog, which is built on the co-addition of all the single frames, is $0.934 \pm 0.036$. Such a value is not available for NEOWISE-R, but the mean of the single-frame measurements is $0.947 \pm 0.118$, where the error corresponds to the dispersion of all individual measurements. Additionally, Figure 3 shows the light curve of the event in the GALEX NUV band and in the Pan-STARRS gri bands. The transient event is clearly observed in all four bands presented.

In order to isolate the emission of the transient event, we need to remove the unrelated underlying emission. This is complicated by the fact that W0948+0318 is a type $1 \mathrm{AGN}$, which are intrinsically variable objects. Indeed, the $V_{\mathrm{CSS}}$-band light curve in Figure 3 shows clear variability, albeit with much lower amplitude, before and after the transient event. This is also observable in the GALEX and Pan-STARRS observations, although much less clearly owing to the fewer number of observations. To isolate the transient in the $V_{\text {CSS }}$ band, we model the AGN variability of W0948+0318 as a DRW using the code JAVELIN ( $\mathrm{Zu}$ et al. 2013). We only use the emission outside of the transient event, specifically at MJD $<54,750$ and MJD $>55,650$. The DRW process is modeled by two quantities: the asymptotic amplitude of the structure function, $\mathrm{SF}_{\infty}$, and the characteristic dampening timescale $\tau$ (see $\mathrm{Zu}$ et al. 2013 , for details). Note that if the region during the transient event is included in the modeling, the fit does not converge. Figure 6 shows the best-fit DRW model and the continuumsubtracted light curve of the event. The DRW model of the AGN light curve provides an expected range of dispersion where no data were available to fit the model. The transient event is well above the expected AGN variability (see also discussion in Graham et al. 2017). For the subtracted light curve we have added in quadrature the $1 \sigma$ range of the best-fit model to the error of each data point. Assuming that the magnitude decay is linear with time, we find a best-fit decay rate for the transient event of $0.0059 \pm 0.0010$ mag day $^{-1}$. Assuming the same for the rise, we get a $\sim 3$ times faster brightening rate of $0.019 \pm 0.003 \mathrm{mag} \mathrm{day}^{-1}$. Note that the uncertainties have been obtained from a Monte Carlo process taking into account the uncertainty in the observed fluxes, as well as the uncertainty from the DRW modeling of the underlying emission.

\subsection{Multiwavelength Transient SED}

As little data are available for the Pan-STARRS and GALEX bands, we cannot model these wavelengths using the DRW approach. Instead, we have simply assumed that the shape of the light curve is the same as in the CRTS $V_{\mathrm{CSS}}$-band DRW model, scaled to a different mean flux. This is not unreasonable given that the flux variability in the accretion disk should be replicated at all wavelengths, with a small time delay (e.g., Edelson et al. 2015) and an amplitude that scales with wavelength (e.g., MacLeod et al. 2010). For the black hole mass, Eddington ratio, and bolometric luminosity estimated in Section 3.3, assuming that the latter corresponds to the bolometric luminosity of the accretion disk (although see Gallagher et al. 2007, for details), the radius of a geometrically thin, optically thick accretion disk (Shakura \& Sunyaev 1973), where the thermal radiation peaks at $5000 \AA$, would be $0.26 \mathrm{lt}$ day (Equation (2) of Morgan et al. 2010). Even though real accretion disks may be $\sim 4$ times larger than a thin-disk model (Morgan et al. 2010), the light-travel time within the accretion disk is much shorter than the timescales on which we are interested, so we ignore this potential concern. To account for the fact that the amplitude of the variability is dependent on wavelength, we scale the $\mathrm{SF}_{\infty}$ and $\tau$ parameters of the DRW model according to the results of MacLeod et al. (2010; although see Kozłowski 2017, who suggests that such correlations could be spurious). Specifically, $\mathrm{SF}_{\infty} \propto \lambda_{\mathrm{RF}}^{-0.479}$ and $\tau \propto \lambda_{\mathrm{RF}}^{0.17}$, where $\lambda_{\mathrm{RF}}$ is the rest-frame effective wavelength of each band to scale from the $V_{\text {CSS }}$ light-curve model.

The same approach is not possible for the WISE bands, as the coverage of the light curve is much more sparse, and outside the transient event, the mid-IR emission is dominated by the dust torus, not by the accretion disk. Instead, we consider that little variability is observed during the NEOWISE-R observations, so we simply subtract their median flux from the AllWISE observations. As the NEOWISE-R epochs were observed starting $\sim 3.5 \mathrm{yr}$ after the AllWISE epochs, which is significantly longer than the travel time from the accretion disk to the torus, ${ }^{20}$ we expect the NEOWISE-R emission to be representative of the typical torus luminosity even if the source of the transient was within the accretion disk. Furthermore, from the analysis of Kozłowski et al. (2016), we expect $\lesssim 0.2 \mathrm{mag}$ variability over the $\sim 3-4$ yr time span between the AllWISE and NEOWISE-R observations, which is small compared to the transient's amplitude of $\sim 1$ mag. Comparing the two AllWISE epochs after the subtraction, we find linear magnitude decay rates of $0.0050 \pm 0.0013$ and $0.0045 \pm 0.0016$ mag day $^{-1}$,

\footnotetext{
${ }^{20}$ In Section 3.3 we estimated an AGN bolometric luminosity for W0948 +0318 of $L_{\mathrm{Bol}}=3.6 \times 10^{44} \mathrm{erg} \mathrm{s}^{-1}$, although, as discussed, this should be considered an upper bound. Using Equation (1) of Nenkova et al. (2008) and assuming a dust sublimation temperature of $1500 \mathrm{~K}$, we estimate the lighttravel time to the inner edge of the torus to be $<0.8 \mathrm{yr}$, as $L_{\mathrm{Bol}}$ is an upper bound on the accretion disk luminosity.
} 


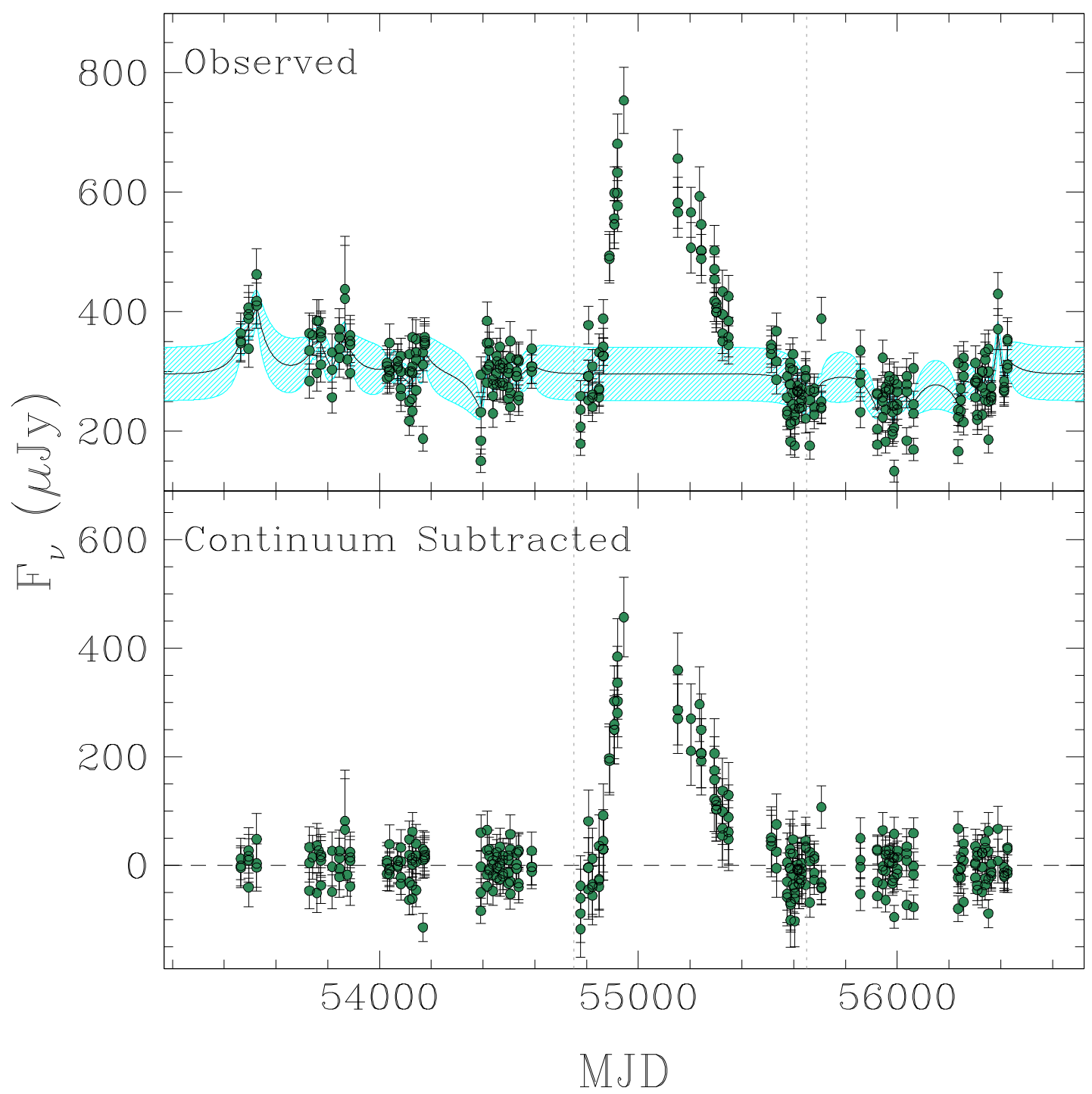

Figure 6. Top: CRTS $V_{\text {CSS }}$-band light curve of W0948+0318. The black solid line shows the best-fit DRW model to the light curve outside the transient event. The cyan region shows the dispersion of the DRW light curves consistent with the data. The vertical dotted lines show the region of the light curve excised for fitting the DRW model. Bottom: $V_{\mathrm{CSS}}$-band light curve after subtracting the best-fit DRW model.

respectively, in the $\mathrm{W} 1$ and $\mathrm{W} 2$ bands, similar to the $0.0059 \pm 0.0010$ mag day $^{-1}$ found for the $V_{\mathrm{CSS}}$ band.

Figure 7 shows the continuum-subtracted specific luminosity of the transient in the UV and optical bands for two epochs. The left panels show MJD 55,230, while the right panels show MJD 55,330, 100 days later. MJD 55,230 was chosen to be close to the first epoch of Pan-STARRS observations and to the second epoch of the NUV observations, thereby minimizing interpolation. To predict the observed flux at each date for each band, we assume the same rate of decay observed for the $V_{\mathrm{CSS}}$-band light curve after the subtraction of the AGN emission. The apparent inconsistency observed between the CRTS and the Pan-STARRS bands is most likely due to the shape of the SED since the CRTS observations are unfiltered. The second date shown in Figure 7, MJD 55,330, corresponds to the first epoch of the WISE observations. Note that the NUV and Pan-STARRS bands shown here are estimated in the same manner as for the MJD 55,230 date. As there are no additional data, these estimates are strongly dominated by the assumption of the decay rate being consistent with that of the $V_{\text {CSS }}$ band.

In the first date for which the SED is shown, MJD 55,230, the NUV, $g$, and $r$ bands are qualitatively consistent with a blackbody whose peak lies between the NUV and $g$ bands. The $i$-band specific luminosity is much higher than would be expected for such a blackbody, but could be driven by the $\mathrm{H} \alpha$ emission line. If we assume that the underlying SED is composed by a blackbody continuum with strong $\mathrm{H} \alpha$ and $\mathrm{H} \beta$ emission lines, we can fit all four data points perfectly, as the model has exactly four parameters. For the emission lines, we assume a Gaussian shape with the same width measured in the SDSS spectrum (i.e., $\sigma=886.5 \mathrm{~km} \mathrm{~s}^{-1}$ ). The best-fit model is shown in the top left panel of Figure 7. For the later date, MJD 55,330, shown in the top right panel, we simply scale the fit to the MJD 55,230 data by the respective NUV specific luminosities. The best-fit blackbody has a temperature of 13,600 $\pm 500 \mathrm{~K}$, and the $\mathrm{H} \alpha$ and $\mathrm{H} \beta$ emission lines have rest-frame equivalent widths (EWs) of $960 \pm 340 \AA$ 


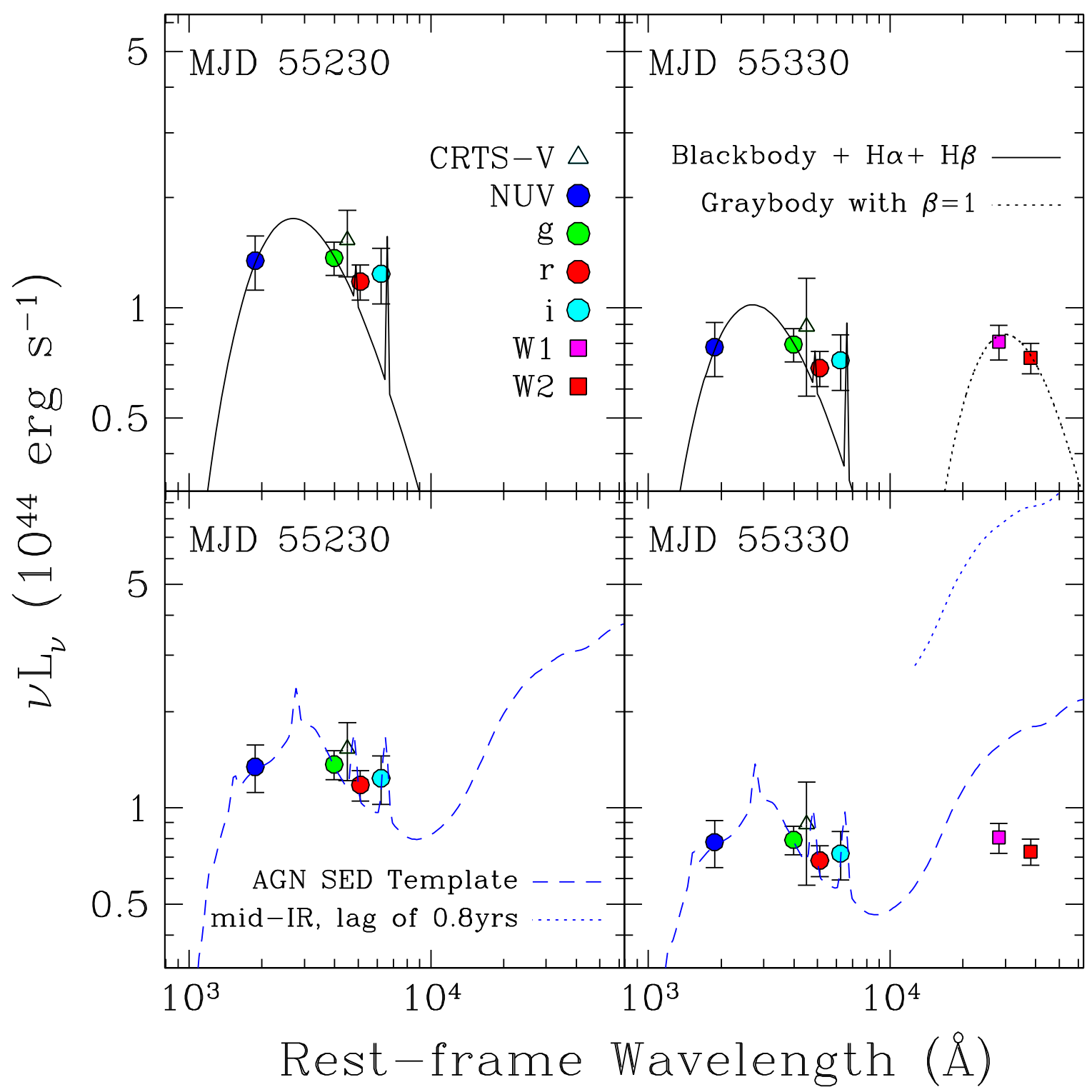

Figure 7. SED of the transient in W0948+0318 at MJD 55,230 (left panels) and 55,330 (right panels). The top panels show the best-fit blackbody and emission-line model (solid black lines) to the optical and UV bands, as discussed in the text. The dashed gray line shows the best-fit modified blackbody (or graybody ) with $\beta=1$ to the WISE W1 and W2 bands. The bottom panels show the best-fit AGN SED model to the data (dashed blue line) for each epoch. In the bottom right panel, the dotted blue line shows the expected mid-IR emission if the transient had occurred within the accretion disk and the light-travel time to the inner edge of the dust torus was $0.8 \mathrm{yr}$, the upper limit we estimate for it.

and $240 \pm 90 \AA$, respectively. Note that since these are broadbands, the EW is very insensitive to the width assumed for the emission lines. For example, if we assume an FWHM of $5000 \mathrm{~km} \mathrm{~s}^{-1}$, the EWs change by less than $1 \%$. Note that all the uncertainties on the best-fit parameters are determined from a Monte Carlo process that considers the uncertainty on the observed fluxes and the uncertainty in the continuum subtraction. Note also that the emission of the WISE bands is inconsistent with this blackbody model, requiring a different component to describe them. We model the WISE bands by a modified blackbody (or graybody) with $\beta=1$ and find an implied temperature of $970 \mathrm{~K}$. The fit is shown in Figure 7, but we note that the temperature is highly unconstrained owing to the fact that we fit two parameters of the model (amplitude and temperature) to two data points.

Alternatively, we could assume that the underlying SED of the source is still that of an AGN. While the analysis of Graham et al.
(2017) determined that the source of the transient cannot be regular AGN variability, it could be the case that the transient event maintains the AGN SED (we discuss this further in Section 5.2). The bottom panels of Figure 7 show the best fit obtained in this case for the two epochs using the AGN SED template and algorithm of Assef et al. (2010), without a host galaxy component. The fit to the UV and optical bands has a $\chi_{\nu}^{2}=0.07$ on both dates. The best-fit model requires a reddening of $E(B-V)=$ $0.16 \pm 0.01$, which is considerably larger than that found for the pre-transient SED of W0948+0318. While this could be taken as an indication that the SED of the transient deviates from that of the AGN in W0948+0318, it is also possible that the nuclear obscuration could have changed between the two epochs.

The best-fit SED at MJD 55,330 significantly overpredicts the observed W1 and W2 fluxes, which is difficult to reconcile with the source of the transient being within the accretion disk. As the emission of the dust torus would simply be reprocessed 
$\mathrm{UV} /$ optical emission from the accretion disk and the transient in this scenario, the excess dust emission would have to be commensurate with the transient emission with a time lag given by the light-travel time to the inner edge of the torus (which is $<0.8 \mathrm{yr}$ ). We estimate that $0.8 \mathrm{yr}$ before MJD 55,330 the $V_{\mathrm{CSS}}$ emission of the transient would be 4.9 times brighter. For comparison, the bottom right panel of Figure 7 shows the expected dust emission reprocessing 4.9 times brighter UV/ optical emission. If the transient had been located within the accretion disk, we would have expected the W1 and W2 fluxes to have been within the two curves shown. That the observed dust emission is significantly fainter instead implies that this physical scenario is not viable.

\subsection{Energy of the Transient Event}

As discussed in the previous section, the $V_{\mathrm{CSS}}$ band and the WISE W1 and W2 bands show different behaviors with time, with the WISE bands fading less quickly than the optical light. This suggests that the WISE bands are tracing a different physical component of the transient. Since the mid-IR emission is likely related to a dusty component that reprocesses the optical emission, we estimate the energy of the transient event by using the $V_{\mathrm{CSS}}$-band light curve alone. Because the light curve is somewhat noisy and sparse, we group the $V_{\mathrm{CSS}}$-band data in bins of 10 days, and we linearly interpolate the binned light curve in magnitude space to determine the luminosity as a function of time.

We first assume the best-fit blackbody discussed in the previous section. Note that while that model also considered strong $\mathrm{H} \alpha$ and $\mathrm{H} \beta$ emission lines, we do not count their contribution to the luminosity for the energy estimate. In order to estimate the total radiated energy, we scale the luminosity of the best-fit model to match the flux at a given date, but we do not allow the temperature to evolve with time. Since the exact filter curve of the $V_{\mathrm{CSS}}$ band is not known, we rely on the empirical calibrations of Drake et al. (2013) between $B, V, R$, and $I$ and the $V_{\text {CSS }}$ band ${ }^{21}$ to estimate the blackbody luminosity at a given time. In practice, we use the Pysynphot v0.9. $7^{22}$ package to compute the observed $B, V, R$, and $I$ magnitudes for the SED model described, and we use these calibrations to estimate its $V_{\mathrm{CSS}}$-band magnitude, and then scale its luminosity to fit the observations. Note that for each set of BVRI magnitudes, the calibrations of Drake et al. (2013) naturally yield three estimates of the $V_{\mathrm{CSS}}$ band and hence three estimates of the luminosity. Considering this, as well as the uncertainties in the best-fit parameters of the SED model and the uncertainties in the DRW subtraction of the underlying continuum, we estimate the total radiated energy of the transient event to be $E=1.6 \pm 0.3 \times 10^{52} \mathrm{erg}$.

As discussed in the previous section, the SED of the transient is somewhat consistent with that of an AGN. If, instead of using the blackbody SED model, we obtain the luminosity by integrating over the best-fit AGN SED model and repeat the above process, we estimate a total radiated energy of $E=1.0 \pm 0.2 \times 10^{53} \mathrm{erg}$. However, in order for this estimate to be accurate, we need to remove the contribution of the dust torus to the luminosity. Assuming that it accounts for about half

\footnotetext{
21 A typographical error is present in Equation (3) of Drake et al. (2013). The correct equation is $V=V_{\mathrm{CSS}}+1.07 \times(R-I)^{2}+0.04$ (A. J. Drake, private communication).

22 https://pysynphot.readthedocs.io/en/latest/
}

of the integrated luminosity of the SED template (after removing the reddening), the energy estimate would be $\sim 5 \times 10^{52}$ erg.

\section{Source of the Transient Event}

Graham et al. (2017) discussed a number of sources similar to W0948+0318 found in the CRTS survey. They explored in detail whether these transients could be microlensing events caused by stars in the Milky Way, SLSNe, TDEs, or black hole binary mergers. Graham et al. (2017) find that the majority of the CRTS transients are incompatible with microlensing events and, as discussed earlier, microlensing of the central engine in W0948+0318 would not be observable in the WISE bands, given that the physical scale of the dust torus is much bigger than that of the accretion disk. Hence, we do not discuss this scenario any further. Below, we first compare the light curve of the transient in W0948+0318 with those of other energetic transient events, and then we discuss its nature in the context of other cases outlined by Graham et al. (2017). We do not discuss stellar mass binary black hole mergers within the accretion disk of the AGN, as, although intriguing, no testable predictions have been made. Additionally, we discuss a mechanism recently proposed by Moriya et al. (2017) to explain similar transients in AGNs.

\subsection{Comparison with Other Energetic Transient Events}

Figure 8 compares the light curve of the transient event in W0948+0318 with a number of very luminous transients in the literature. The figure shows the light curve for the luminous transient event CSS 100217 discovered by Drake et al. (2011) using CRTS, with a total radiated energy of $1.3 \times 10^{52} \mathrm{erg}$. The peak absolute $V_{\mathrm{CSS}}$-band magnitude of this event is similar to that of the one in W0948+0318, although it has a faster decay rate. Interestingly, the transient occurred in a narrow-line Seyfert 1 galaxy, similar to the host of W0948+0318 (see Section 3.3). Drake et al. (2011) discuss a number of scenarios for this transient and find that the most likely is that CSS 100217 corresponds to a Type IIn SN that occurred within the narrow-line region of the AGN. We also show the luminous transient event PS16dtm (or SN 2016ezh), also hosted in an AGN (specifically a narrow-line Seyfert 1). Blanchard et al. (2017) show that the light curve of this object is very flat in a number of different optical bands, and they determine that the transient is best modeled by a TDE. The $g$-band light curve of the significantly less luminous TDE PS10jh (Gezari et al. 2012) is also shown. Unlike the previous objects, PS10jh has a significantly faster decay time, and it is not hosted in an AGN.

Figure 8 also shows the $V$-band light curve of ASASSN15lh. Discovered by Dong et al. (2016), this is the most luminous SN ever detected, with a total radiated energy of $(1.7-1.9) \times 10^{52} \mathrm{erg}$ within its first 550 days (Godoy-Rivera et al. 2017). Dong et al. (2016) determined this transient to be a hydrogen-poor SLSN (although see Leloudas et al. 2016, who have suggested this transient to be a TDE from a Kerr black hole). The decay rate of this event is quite similar to that of the transient in W0948+0318 and likely has a similar peak magnitude. Additionally, the figure shows the $R$-band light curve of the luminous hydrogen-rich supernova SN 2006gy from Smith et al. (2007), for which they determined a total radiated energy of $1.2 \times 10^{51} \mathrm{erg}$. The decay rate of this transient is much faster than that of the transient in W0948+0318, 


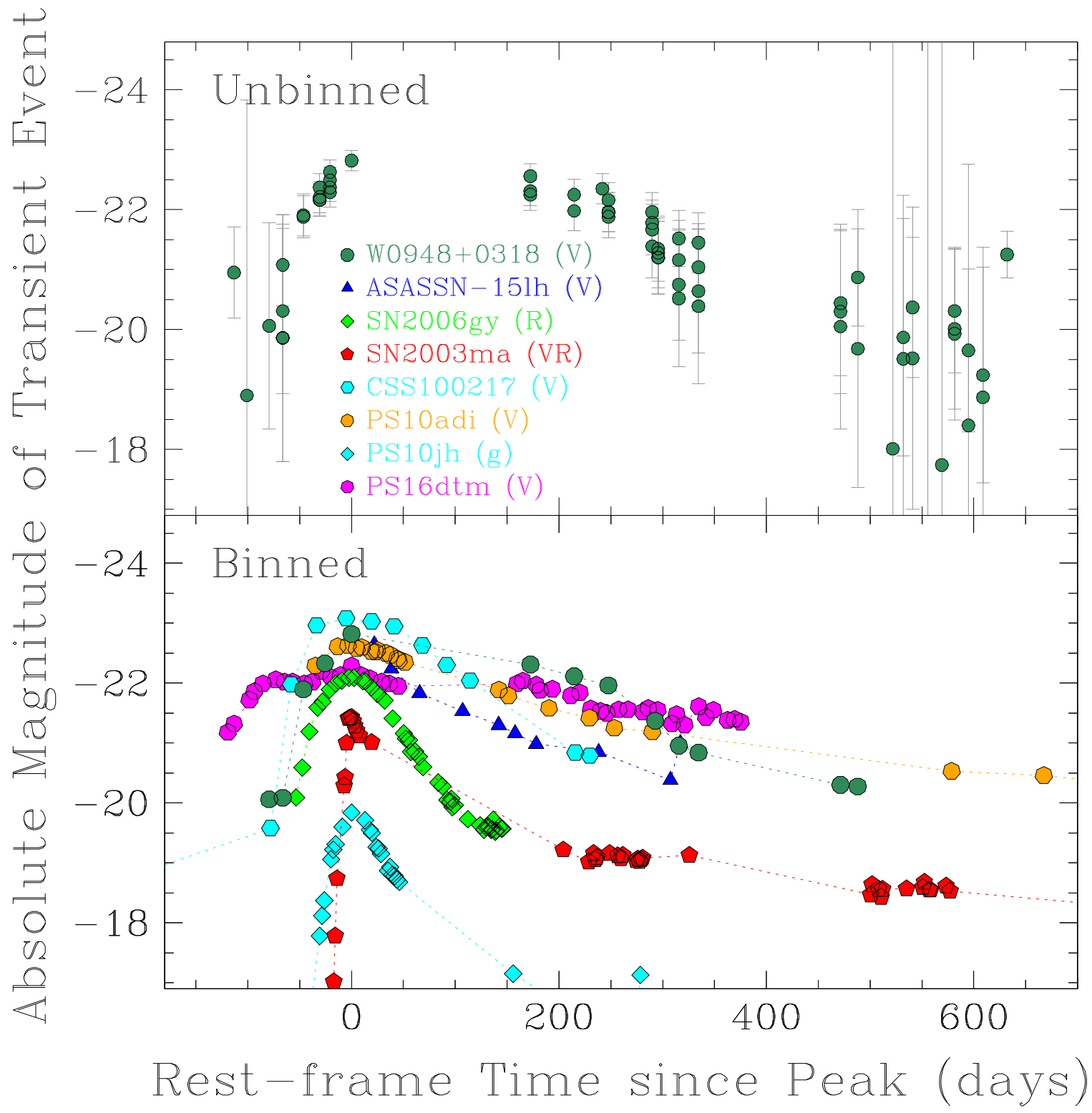

Figure 8. Top: green circles show the CRTS $V_{\mathrm{CSS}}$-band light curve of the transient in W0948+0318. Bottom: green circles show the same as in the top panel, but binned in groups of 10 days. The figure also shows a number of different luminous transients in the literature for comparison with the transient in W0948+0318.

suggesting a different physical origin. A different luminous Type IIn SN, SN 2003ma (Rest et al. 2011), is also shown, and while it has a significantly lower peak magnitude than either SN 2006gy or W0948+0318, it has a slower decay rate, similar to that observed for the transient in W0948+0318. Rest et al. (2011) estimate the total radiated energy of $\mathrm{SN} 2003 \mathrm{ma}$ to be $(3.6 \pm 1) \times 10^{51} \mathrm{erg}$.

Recently, Kankare et al. (2017) studied the very luminous transient PS10adi, whose host galaxy is an AGN. The total energy radiated by the transient is estimated to be $\sim 2.3 \times 10^{52} \mathrm{erg}$, somewhat above, but comparable to, the energy we estimated for the transient in W0948+0318. Kankare et al. (2017) concluded that this transient was consistent with a TDE or a superluminous Type II SN within the narrow-line region of the AGN. The light curve of PS10adi is also shown in Figure 8, and it can be seen to be very similar to that of the transient in W0948+0318, suggesting a common origin. In fact, Kankare et al. (2017) identified W0948+0318 as a possible analog of PS10adi. In the next sections we will discuss the possible sources for the transient in W0948+0318 and find that it is unlikely for the transient to be a TDE or a Type II SN within the accretion disk of the system, instead favoring a location within the dust torus of the AGN.

\subsection{Superluminous Supernova}

Given the high peak luminosity $\left(M_{V} \sim-23\right)$ and long-lasting light curve, if the transient in W0948+0318 was due to an SN, it would have to be an SLSN (Gal-Yam 2012). As discussed earlier, the superluminous SNe ASASSN-15lh (hydrogen poor), SN 2003ma, and CSS 100217 (hydrogen rich) all show comparably slow (albeit slightly quicker) decay rates to the transient in W0948+0318. If the source of the transient in W0948+0318 were an SLSN, we would expect that the underlying SED would be generally consistent with a blackbody. As discussed in Section 4.3, the SED of the transient in W0948 +0318 is indeed consistent with a blackbody, as long as we allow for significant $\mathrm{H} \alpha$ and $\mathrm{H} \beta$ emission, which is also expected for 
SNe in general. Assuming this SED, in Section 4.4 we found a total radiated energy of $E=(1.6 \pm 0.3) \times 10^{52} \mathrm{erg}$. This is consistent with the total radiated energy of $1.3 \times 10^{52} \mathrm{erg}$ estimated for CSS 100217 by Drake et al. (2011), but significantly larger than the $3.6 \times 10^{51} \mathrm{erg}$ estimated for $\mathrm{SN}$ 2003ma by Rest et al. (2011).

The total radiated energy for ASASSN-15lh was estimated by Godoy-Rivera et al. (2017) to be $(1.7-1.9) \times 10^{52} \mathrm{erg}$ within the first 550 days after first detection, which is consistent with the value estimated for the transient in W0948+0318. Dong et al. (2016) also found that the best-fit blackbody to ASASSN-15lh had temperatures ranging from $21,000 \mathrm{~K}$ at 15 days after the peak to $13,000 \mathrm{~K}$ at 50 days after the peak. Godoy-Rivera et al. (2017) found that after $\sim 200$ days the event started rebrightening and the temperatures started increasing again with time, from a minimum of $11,000 \mathrm{~K}$ to a maximum of $18,000 \mathrm{~K}$. For the transient in W0948+0318, we found a best-fit blackbody temperature of $13,600 \pm 500 \mathrm{~K}$ at $\sim 250$ days after the peak, consistent with the range found in ASASSN-15lh. We note that our energy estimate assumes a single temperature throughout the duration of the transient. Significantly lower temperatures would not have a major effect on the estimated luminosities at each date, as the bulk emission of such a blackbody would be within the wavelength range covered by the CRTS $V_{\text {CSS }}$ band. On the other hand, significantly higher temperatures would strongly increase the estimated luminosity at each date, since the $V_{\mathrm{CSS}}$ band would only cover the Rayleigh-Jeans tail of the continuum emission. Hence, if the transient in W0948+0318 has the same physical origin as ASASSN-15lh, then our estimate for the total energy radiated would be a conservative lower bound.

As mentioned above, we find that in order to explain the SED we need $\mathrm{H} \alpha$ and $\mathrm{H} \beta$ emission lines with EWs of $960 \pm 340 \AA$ and $240 \pm 90 \AA$, respectively, which are compatible with what has been observed in luminous SNe. For example, Jencson et al. (2016) observed that in the luminous Type II supernova SN 2010j1 the $\mathrm{H} \alpha$ EW varied between 170 and $3500 \AA$ throughout the lifetime of the transient but was approximately constant at $\sim 2000 \AA$ at late times ( $~ 400$ days after first detection), a similar timescale at which we can model the SED of W0948+0318. Jencson et al. (2016) also observed that the $\mathrm{H} \beta$ EW varied between 35 and $300 \AA$.

Hence, we conclude that the transient in W0948+0318 is highly compatible with an SLSN. For hydrogen-poor (or Type I) SLSNe, the transient is thought to be related to the formation of a magnetar during an SN explosion, where the increased luminosity arises from the coupling between the magnetar spin-down energy and the SN ejecta (Kasen \& Bildsten 2010; Woosley 2010). For Type II (hydrogen-rich) SLSNe the leading mechanism for its radiated energy, instead, is thought to come from the interaction of an $\mathrm{SN}$ explosion ejecta with a dense circumstellar medium (CSM; e.g., see Smith et al. 2007; Smith 2014). Such a dense CSM could naturally be found within or in the vicinity of an AGN. In fact, Sukhbold \& Woosley (2016) argue that total radiated energies in excess of $\sim 3 \times$ $10^{51}$ erg are not attainable by Type II SLSNe if the CSM only comes from material ejected by the parent star prior to the explosion. Furthermore, the large amount of hot dust emission observed during the transient in W0948+0318 suggests that the transient occurred within a very dusty medium, which could be consistent with the dust torus of the AGN. As discussed in
Section 4.3 , it is unlikely that the SLSN would be within the accretion disk, as the W1 and W2 magnitudes do not seem to lag the optical magnitudes with a long enough timescale. However, the fact that we see little to no evolution in the W1-W2 color throughout the transient, and at later times, implies that the dust is not changing temperature, and could be consistent with the transient occurring within the torus, as the accretion disk emission would maintain the high temperature regardless of the transient luminosity. The high gas density in the torus could provide an ideal CSM to generate high luminosities.

We note that Drake et al. (2011) came to a similar conclusion for CSS 100217, i.e., an SN within the immediacies of the AGN. The similarities in energy and light curve suggest that they may have a similar origin, but unfortunately there were no time-resolved mid-IR observations of the CSS 100217 transient to compare with the optical light curve.

\subsection{Accretion Disk Flares, TDEs, and Accretion Disk Outflows}

As discussed in Section 4.3, the WISE light curves are inconsistent with the transient occurring in the accretion disk of the AGN, which in turn implies that the transient in W0948 +0318 is unlikely related to a TDE. Furthermore, the optical SED itself also suggests that a TDE is an unlikely source for the transient. While the majority of TDE events identified in the literature have significantly lower peak luminosities than the transient in W0948+0318 (e.g., Gezari et al. 2012; Holoien et al. 2014, 2016a, 2016b; Brown et al. 2018), these have all been in galaxies that do not host nuclear activity, implying that there is significantly less material for the ejecta to interact with and hence increase the total radiated energy output. However, recently Blanchard et al. (2017) identified the TDE candidate PS16dtm, whose host galaxy has an AGN similar to that of W0948+0318. This TDE is significantly more luminous than all previous TDE candidates. While the peak magnitude of this event is $\sim 1$ mag fainter than the transient in W0948+0318, the event is extremely energetic, owing in great part to its almost constant light curve $\sim 100$ days after peak emission. Unfortunately, there is a gap of $\sim 150$ days after the peak in the CRTS observations of W0948+0318, so we cannot fully assess the similarity of the light curves in this period. Yet, the light curve of the transient in W0948+0318 seems to be highly inconsistent with such behavior in Figure 8. We note that Kankare et al. (2017) find that PS16dtm is a possible analog to PS10adi, just as they find for W0948+0318. We also note that recently Mattila et al. (2018) observed a dust-obscured TDE in the nucleus of the nearby galaxy Arp 299, with an estimated total radiated energy $>1.5 \times 10^{52} \mathrm{erg}$, and likely in the range $(1.9-6.5) \times 10^{52}$ erg when considering dust re-radiation at longer IR wavelengths not covered by their observations. This is somewhat larger than, yet comparable to, the total radiated energy estimated for the transient in W0948+0318. However, the Spitzer/IRAC observations of the event in the [4.5] band show that the brightness of the transient falls approximately $0.9 \mathrm{mag}$ in the 2000 days after the peak (or $4.6 \times 10^{-4} \mathrm{mag} \mathrm{day}^{-1}$ ), an order of magnitude more slowly than found for the W2 band light curve of the transient in W0948+0318 in Section 4.3, suggesting that they might be powered by different mechanisms.

Recently Moriya et al. (2017) proposed a mechanism to explain transients in AGNs such as CSS 100217 and PS16dtm, through the interaction of mass ejections from the accretion 
disk instead of unrelated explosive phenomena such as $\mathrm{SNe}$ or TDEs. Specifically, they ascribe the observed transients to the interaction between mass outflows from the accretion disk and the BLR. They determined that a mass outflow of $1 M_{\odot}$ can produce a total radiated energy of $10^{52} \mathrm{erg}$ over timescales of a few hundred days. Unfortunately no specific predictions are made about the light curve, and hence it is difficult to test how well the transient in W0948+0318 can be described by this model. In the specific case of CSS 100217, Moriya et al. (2017) describe that these transients may be reoccurring in timescales of years to decades. Given the similarities between the two cases, continuous monitoring of this source would be necessary to confirm this scenario. However, as the radius of the BLR, $R_{\mathrm{BLR}}$, in W0948+0318 would be at $\sim 25$ lt-day from the accretion disk according to the AGN's $5100 \AA$ luminosity and the $R_{\mathrm{BLR}}-L_{5100}$ relation of Bentz et al. (2009), the low mid-IR emission compared to the optical emission observed during the transient (see Section 4.3) may be hard to reconcile with this scenario.

\section{Obscured Analogs to W0948+0318}

As discussed in Sections 4.2 and 4.4, the mid-IR emission is likely tracing a different physical component than the optical emission. This component is likely a dusty structure reprocessing the optical light emission of the transient. This structure could be either the dust torus if the source of the event was the accretion disk itself or a star close to it, or circumstellar dust in the case in which the source was an SN in the host galaxy. In Section 2.2 we showed that none of the other optical light curves for the objects in our sample are qualitatively similar to that of W0948+0318. However, because of the nearby dust reprocessing the optical emission, it is possible that analogs to W0948+0318 exist within our sample but are simply dust obscured.

We search for transients with similar WISE light curves to W0948+0318 among our sample. In order to identify the candidates, we use the following criteria, based on the light curve of W0948+0318:

a. The first $2 \mathrm{yr}$ of the NEOWISE-R data should not be highly variable. Specifically, we require that a constant flux fit to the NEOWISE-R data yields a reduced $\chi^{2}$ of $<3$ within its first four epochs. We do not extend this to the later data, as AGNs are intrinsically variable in the mid-IR in long timescales (e.g., Kozłowski et al. 2016).

b. The light curve must show excess flux during the AllWISE observations as compared to the NEOWISE-R ones. In practice, we require that the median magnitude of the last AllWISE epoch is brighter than the median magnitude of the first NEOWISE-R epoch.

These criteria result in 12 candidates, in addition to W0948 +0318 , out of the sample of 45 highly variable mid-IR AGNs identified by Assef et al. (2018). These 12 candidates are shown in Figures 9 and 10. Most of the objects show mid-IR light curves that are highly compatible with that of W0948 +0318 , although none of them show any significant optical variability in CRTS. If these transients have the same origin as in W0948+0318, then it is further unlikely that the accretion disk is the source of the transient, as its optical emission would have been detected by CRTS in at least the two sources that are spectrally classified as QSOs by SDSS, namely, WISE J130716.98+450645.3 $(z=0.084)$ and WISE J161846.37 $+510035.2(z=0.319)$. In those objects we have a direct line of sight toward the accretion disk that is not obscured by the dust torus, unless they have significantly changed their optical obscuration since when the spectroscopic observations were obtained. In order to test this possibility, spectroscopy of the reminder of the sample is being gathered, and those will be later reported by H. Jun et al. (2018, in preparation).

If we apply the same selection criteria to the sample of Graham et al. (2017) discussed in Section 2.2 and further request that the AllWISE observations have var_flg $=9$ in both W1 and W2, we find that W0948+0318 is the only source selected. If we relax the latter requirement to only require var_flg $\geqslant 7$ in both bands, i.e., that the source is a real variable in W1 and W2 during AllWISE, we find that one additional source, CRTS $0234+0107$, would be selected as an analog to W0948+0318. The optical and mid-IR light curve of this source, along with those of the rest of the Graham et al. (2017) sample, is shown in Appendix C.

In Section 4.2 we discussed that the W1-W2 color of W0948 +0318 did not change between the AllWISE and NEOWISE-R observations and was consistent with the AGN classification of Assef et al. (2018) at all times. In Figure 11 we show the W2 magnitude and W1-W2 color of each AllWISE epoch and the mean of the NEOWISE-R epochs. We also show the R90 AGN selection criteria of Assef et al. (2018), as well as their less restrictive (but lower reliability) R75 selection. All objects redder than the R90 or R75 boundaries are considered to be AGNs. During the AllWISE epochs, all objects are consistent with the R90 classification at least within the error bars, although some show a clear transition into bluer, and hence less AGN dominated, mid-IR SEDs. For the NEOWISE-R observations, however, there are four objects significantly bluer than the R90 criteria. Two of these are consistent, at least within the error bars, with the R75 criteria and hence might still be bona fide AGNs. The other two would certainly not be classifiable as AGNs: WISE J130716.98+450645.3 (W1307 +4506) and WISE J130819.12+434525.6 (W1308+4345). Notably, both have spectroscopic observations from SDSS, and both are classified as AGNs. Specifically, W1307+4506 is classified as a QSO at $z=0.084$ with clear broad $\mathrm{H} \alpha$ and $\mathrm{H} \beta$ profiles, while $\mathrm{W} 1308+4345$ has narrow-line ratios that imply that it is a type 2 AGN. The change in WISE colors implies that while during the AllWISE observations the W1 and W2 bands were dominated by the hot dust emission from the AGN torus, in the NEOWISE-R observations they are dominated by the stellar emission from the host galaxy. Hence, the AGNs in these objects were caught by the AllWISE observations possibly migrating into a lower accretion state and could be classified as changing-look AGNs. Indeed, Assef et al. (2018) classified WISEA J142846.71+172353, one of the other highvariability WISE AGN candidates (although not an analog to W0948+0318), as a changing-look quasar. Follow-up spectroscopic observations to be presented by H. Jun et al. (2018, in preparation) will be able to better constrain the nature of these two targets.

$\mathrm{W} 1308+4345$ is one of three of the possible analogs to W0948+0318 objects that have recently been identified as possible TDE candidates by Wang et al. (2018). The other two objects are WISEA J091225.00+061014.8 (W0912+0610) and WISEA J100933.13+232255.7 (W1009+2322). We note that W1009+2322 and W1308+4345 are classified as "Galaxy AGN" by SDSS, although Wang et al. (2018) instead classify 


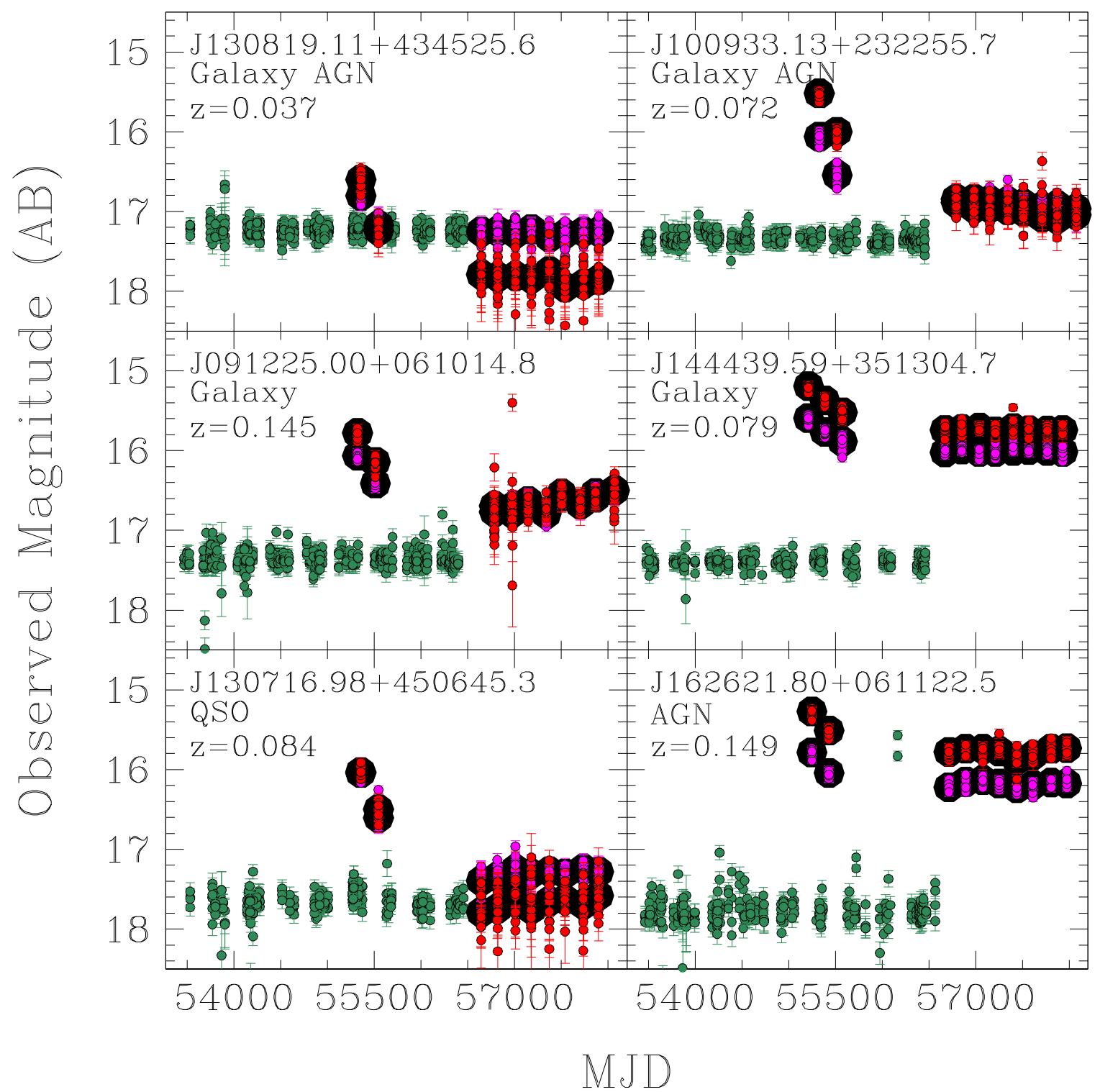

Figure 9. Light curves of the six brightest analogs to W0948+0318 identified in Section 6. The sources are arranged in descending brightness order. The symbols have the same meaning as in Figure 2.

them as star-forming galaxies. W0912+0610 is identified by SDSS as a galaxy without an AGN component. If these objects are indeed TDEs, then they are unlikely analogs to W0948+0318.

For the remaining targets, there are a number that are classified as type 1 AGNs or QSOs that do not show any optical variability associated with the mid-IR transient. In the previous section, we concluded that the most likely explanation for the transient in W0948+0318 is an SLSN, and we speculated that, as a result of the near-constant mid-IR color throughout and after the transient event, the SLSN could have happened within the torus of the AGN, and that we happen to have an unobscured line of sight toward the event. If we assume that the majority of these analogs share a similar origin to the transient in W0948+0318, the lack of optical transients is consistent with locations within the tori of their AGNs. In other words, in the majority of these events, the transient would remain obscured and only observable in the mid-IR, with the transient in W0948+0318 being the exception on which an unobscured line of sight was available.

\subsection{Rates}

Determining an event rate is difficult in this case as we have very little data to characterize the transient, and there are a large number of selection effects that went into the identification of W0948+0318. The parent sample consisted of 4,544,197 AGN candidates from the R90 catalog of Assef et al. (2018), which considers all selected W1 and W2 AGNs in 30,093 deg ${ }^{2}$ of the AllWISE data release. This area corresponds to the entire extragalactic sky, avoiding the Galactic center, the Galactic plane, nearby galaxies, and known star formation regions in our Galaxy (see Assef et al. 2018, for details). Assef et al. (2018) required all their candidates to be point sources in the WISE images, which puts a significant bias against the most nearby objects.

Furthermore, the selection of variable objects required them to be identified with the highest variability level in the AllWISE source catalog. These criteria are based on the probability of the source being variable given its fluxes and uncertainties in the single-frame observations and generates 


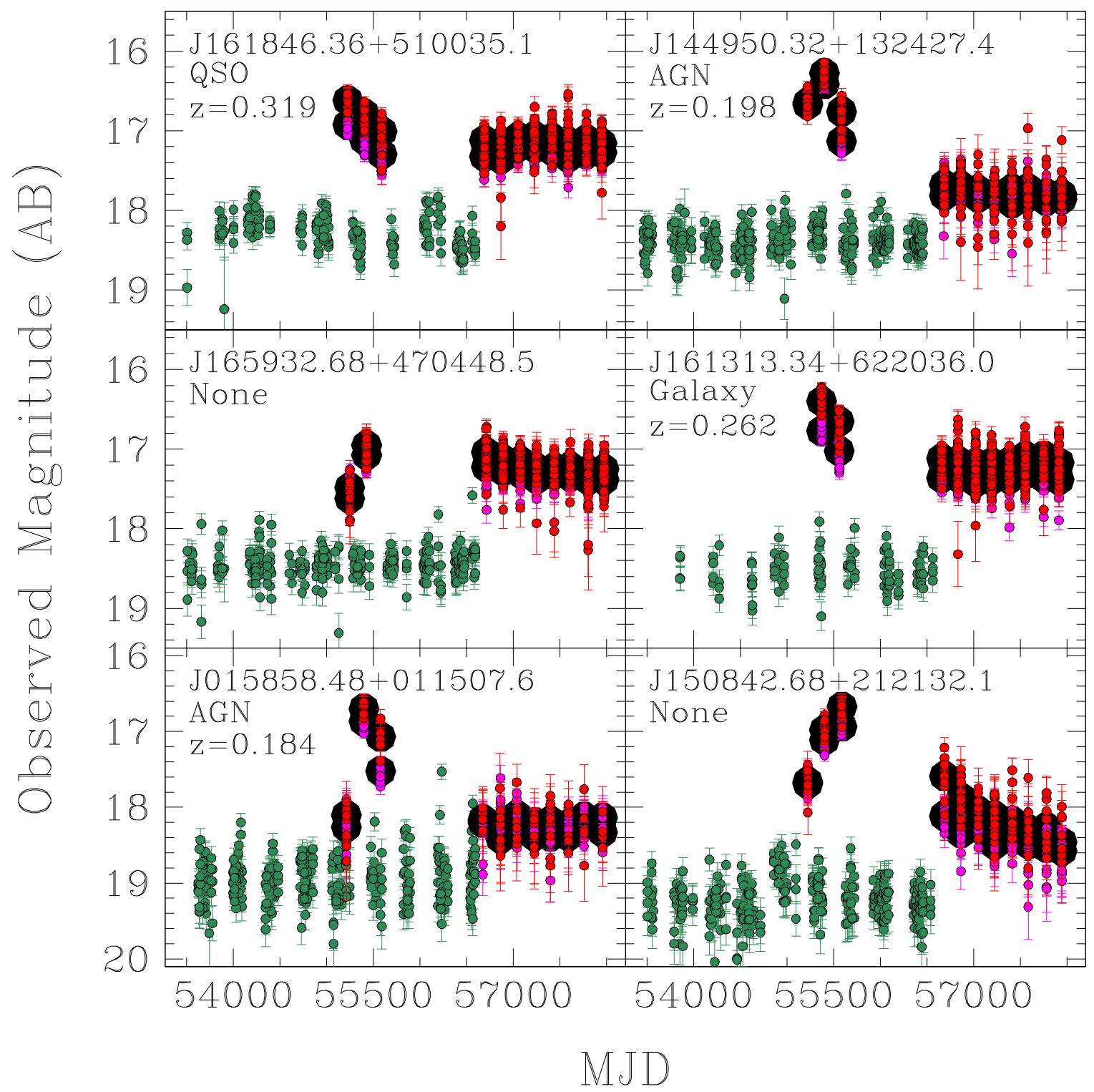

Figure 10. Light curves of the six faintest analogs to W0948+0318 identified in Section 6 . The sources are arranged in descending brightness order. The symbols have the same meaning as in Figure 2.

two biases. The first is that the number of single-frame observations is a steep function of the ecliptic latitude due to the survey scan pattern of WISE. This means that sources at higher ecliptic latitudes have better statistics from which to compute this variability index. The second bias is against fainter objects, as their single exposure photometric uncertainties are larger, requiring a higher amplitude of the variability to be cataloged with the highest variability level. In fact, W0948 +0318 has co-added magnitudes in AllWISE of W1 $=13.537$ and $\mathrm{W} 2=12.603$, while the median magnitudes of the R90 catalog of Assef et al. (2018) are 16.58 and 15.42, respectively. Only $0.4 \%(18,823)$ of the sources are brighter than W0948 +0318 in W1, and only $0.6 \%(28,242)$ are brighter in W2. To have a good variability detection, the sources have to be well detected in the single-frame exposures, which requires $\mathrm{W} 1<14.25$ mag. ${ }^{23}$ For fainter W1 magnitudes the variability detection is severely degraded. In fact, of all the analogs of W0948+0318 identified in the previous section, only WISEA

\footnotetext{
${ }^{23}$ http://wise2.ipac.caltech.edu/docs/release/allwise/expsup/sec2_3c.html
}

J165932.68+470448.5 has a peak magnitude fainter than this limit, with a median $\mathrm{W} 1=14.39$ in the brightest AllWISE epoch. Further inspection, however, shows that the peak singleframe magnitude measured for this object is $\mathrm{W} 1=11.56$, with the second-brightest magnitude being $\mathrm{W} 1=14.26$. As the AllWISE database does not show any other targets within $3^{\prime \prime}$ of WISEA J165932.68+470448.5, the assignment of the highestvariability flag could be spurious. Hence, we do not consider this object further in our analysis.

In the NEOWISE-R epochs, the mean magnitude of W0948 +0318 in $\mathrm{W} 1$ is 14.12 , or $\sim 0.77$ mag fainter than the median W1 magnitude in the first, brightest AllWISE epoch. Hence, we can assume that we should have been able to detect a transient like the one in W0948+0318 in any host with an apparent brightness down to 0.77 mag fainter than the $\mathrm{W} 1=14.25 \mathrm{mag}$ limit discussed above, i.e., with $\mathrm{W} 1 \lesssim 15.02 \mathrm{mag}$. In the R90 catalog of AGN candidates of Assef et al. (2018) there are 338,861 such objects, and hence we can use this number to estimate the rate of these transient events. This is a conservative assumption, however, as it is unlikely that the object would 


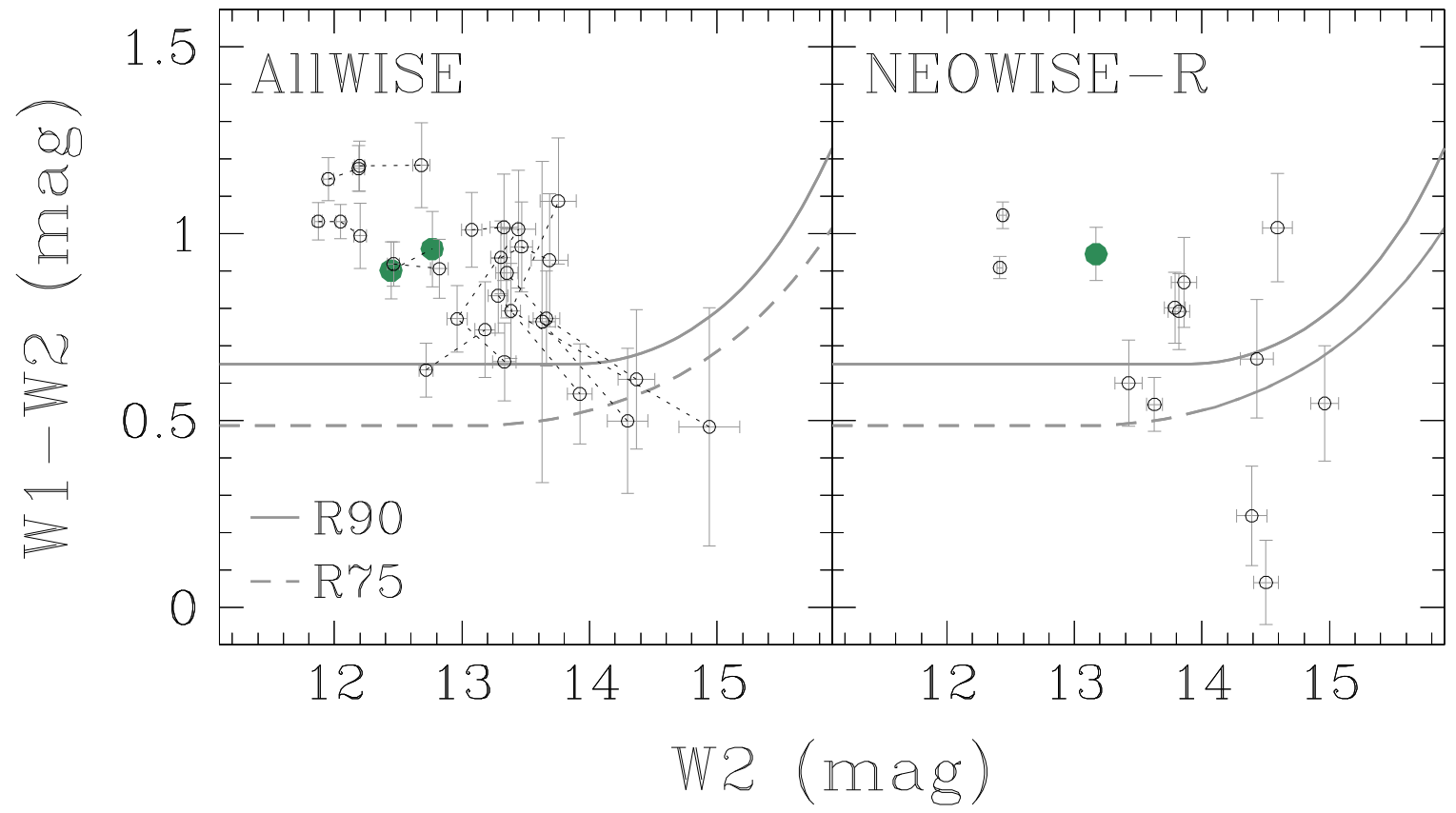

Figure 11. W1-W2 color against W2 magnitude for W0948+0318 (large green circles) and its possible analogs (small open circles). The left panel shows the quantities during each AllWISE epoch, where different epochs are connected by the dotted lines. The right panel shows the average values of these quantities during the NEOWISE-R epochs for each object. In each panel, the solid (dashed) gray line shows the R90 (R75) AGN selection criterion of Assef et al. (2018).

have been identified as a highly variable AGN by WISE so close to the detection limit, in particular without having been detected in more than one epoch.

Considering all of these sources are at low redshifts, and hence to zeroth order we can ignore the cosmological time dilation, we can get a lower bound on the estimate of the occurrence of these transients. The duration of the optical transient in W0948+0318 is $\sim 800$ days, counting from the peak emission to the point where the CRTS light curve becomes dominated by the host emission. Since the AllWISE survey has a span of approximately 180 days between epochs, we probe a total period of $\sim 1000$ days, meaning that we could have detected from transients that started $\sim 800$ days before the first epoch up to transients that started on the second AllWISE epoch. This assumption may oversimplify the estimate, as it implies that we could have detected the transient at any stage of its evolution. Disregarding this issue, we can estimate that the rate per $\mathrm{AGN}$ of these transients would be approximately $1 / 338,861 /(1000$ days $)=1.1_{-0.9}^{+2.5} \times 10^{-6} \mathrm{yr}^{-1}$, or about once in every $900,000_{-640,000}^{+4,400,000} \mathrm{yr}$ in a given AGN if W0948+0318 is a unique object within our sample (i.e., disregarding all possible analogs presented in the previous section). The error bars consider the uncertainty derived from a Poisson distribution (Gehrels 1986). However, because of the simplifications made, this should be considered as a lower bound estimate. This further becomes the case when we consider that for a fixed energy of the transient the effects will be significantly less obvious in more luminous AGNs, and hence we might not see them. We can then only conclude that the detection implies that these transients happen with a rate greater than $>2 \times 10^{-7} \mathrm{yr}^{-1}$ per AGN, or more than once every $\sim 5 \times$ $10^{6} \mathrm{yr}$. Considering that the lifetime of an AGN is $\gtrsim 10^{7} \mathrm{yr}$ (Martini \& Weinberg 2001; Marconi et al. 2004), the above rates suggest that these transient events would occur $\gtrsim 2$ times during the phase on which the central supermassive black hole is actively accreting.
Similarly, we can consider instead the event in isolation, and consider that we could have detected the transient if it was up to a redshift on which the apparent peak magnitude at W1 would have been $14.25 \mathrm{mag}$. Neglecting $K$-corrections, we find that the redshift at which this apparent magnitude would have been observed is $z=0.290$. Considering that the R90 catalog of Assef et al. (2018) covers 30,093 $\mathrm{deg}^{2}$ of the sky, we find that the event was detected within a comoving volume of $5 \mathrm{Gpc}^{3}$. This implies a rate of $0.07_{-0.06}^{+0.17} \mathrm{Gpc}^{-3} \mathrm{yr}^{-1}$. As per the discussion above, this number is likely a lower limit, and hence we conclude that the rate is $>0.01 \mathrm{Gpc}^{-3} \mathrm{yr}^{-1}$. If we compare with the rate of Type II SLSNe of $151_{-82}^{+151}$ events $\mathrm{Gpc}^{-3} \mathrm{yr}^{-1} \mathrm{~h}_{71}^{3}$ found by Quimby et al. (2013), we find that the transients in W0948+0318 are less frequent by a factor of $<151,000$. Quimby et al. (2013) find a rate of Type I SLSNe of $68_{-44}^{+94}$ events $\mathrm{Gpc}^{-3} \mathrm{yr}^{-1} \mathrm{~h}_{71}^{3}$, implying that the rate of transients like the one in W0948+0318 is less frequent by a factor of $<6800$. This implies that we cannot, in principle, disregard the possibility that the transient in W0948+0318 is an SLSN unassociated with the AGN. If this had been the case, then we would have expected that the rates would differ by a factor of $\sim 1000$, as roughly 1 in every 1000 galaxies hosts an active nucleus, which is consistent with the estimates above. However, the transients studied by Quimby et al. (2013) have lower radiated energy than the transient in W0948+0318, and if there is a steep dependence of the rates on energy, as suggested by Godoy-Rivera et al. (2017), the rates could be hard to reconcile.

In the previous section we presented 12 additional objects whose WISE light curves suggest that they could be analogs to W0948+0318, although two of them showed W1-W2 colors during the NEOWISE-R observations no longer consistent with an AGN classification, unlike W0948+0318, and one may have a spurious variability classification. If we assume that the remaining nine transient candidates are obscured analogs to the transient in W0948+0318, their rate per AGN would instead be 
$>7.5 \times 10^{-6} \mathrm{yr}^{-1}$, or greater than once every $135,000 \mathrm{yr}$, about 30 times more frequent. Additionally, we can estimate the event rate density by considering, as done above, the maximum volume in which we could have identified the targets. Excluding WISEA J150842.68+212132.1, for which we do not have a redshift, we find a rate of $>1.2 \mathrm{Gpc}^{-3} \mathrm{yr}^{-1}$. This would imply that these transients are a factor of $<125$ $(<57)$ times less frequent than Type I (II) SLSNe. These numbers are somewhat inconsistent with the estimate of Quimby et al. (2013) if we assume that 1 in every 1000 galaxies hosts an AGN, pointing to a relation between AGN activity and these kinds of transient events, and further supporting the scenario discussed earlier of SLSNe preferentially occurring in the tori of AGNs.

\section{Conclusions}

Assef et al. (2018) recently presented two catalogs of WISEselected AGNs over 30,093 $\mathrm{deg}^{2}$. From their catalog optimized for sample reliability with $\sim 4.5$ million AGN candidates, Assef et al. (2018) noted that 687 were classified with the highest variability level in the AllWISE database. Assef et al. (2018) found that for the 207 of those targets located within the FIRST survey footprint, 162 (78\%) were detected at radio energies, implying that they are most likely blazars. Here, we have presented a detailed study of the 45 highly variable WISE AGN candidates that are undetected by FIRST. Assef et al. (2018) discussed the spectroscopic classification of 34 of these sources, primarily from literature observations. They found that 31 of the 34 targets were consistent with an AGN classification. In Section 2.1 we presented spectra for five additional sources and found four of them to have clear AGN signatures.

Although these 45 sources are highly variable in the WISE bands during the AllWISE mission, we find that only seven show significant optical variability in the CRTS survey, which covers the complete time span of the AllWISE observations (see Section 2.2 for details). Two of them are carbon stars and are contaminants to our AGN sample. The other five have spectroscopic AGN classifications. Four show low-amplitude optical variability, while the fifth one, W0948+0318, shows a very bright optical transient, coincident in time with the AllWISE epochs of observation. W0948+0318 is spectroscopically classified by SDSS as a QSO at $z=0.2073$. From the SDSS optical spectrum, obtained significantly before the transient, we find a supermassive black hole mass of $M_{\mathrm{BH}}=1.2 \pm 0.4 \times 10^{7} M_{\odot}$ and that the AGN in W0948 +0318 had an Eddington ratio at the time of $0.25 \pm 0.14$. From GALEX, SDSS, and 2MASS broadband photometry obtained before the transient event, we find an SED that is best modeled as a type 1 AGN with very little, and possibly no, reddening, but with a significant host component.

In order to isolate the emission from the transient event from that of the AGN itself in the CRTS $V_{\mathrm{CSS}}$ band, we modeled its light curve before and after the transient event with a DRW model and subtracted the mean expected AGN emission during the transient event. We find that the transient event is very asymmetric, with an optical rise time of 0.019 mag day $^{-1}, \sim 3$ times faster than the decay rate of $0.0059 \mathrm{mag}^{\text {day }^{-1}}{ }^{-}$. We used the best-fit DRW model to the $V_{\mathrm{CSS}}$ band to also isolate the transient event emission in multiwavelength broadband observations from GALEX in the NUV band and from Pan-STARRS in the $g, r$, and $i$ bands. To isolate the transient emission in the
WISE bands, we subtracted from the AllWISE bands the median of the NEOWISE-R data, which were obtained significantly after the transient. The multiwavelength SED at two different epochs is shown in Figure 7. We find that if we model the optical bands as an AGN, the WISE bands are significantly overpredicted. This implies that the transient event cannot correspond to a flare in the accretion disk, a TDE, or another kind of transient within the accretion disk, as if that had been the case, the best-fit SED to the optical data would underpredict the emission in the WISE bands instead owing to the light-travel time from the accretion disk to the dust torus. We also find that the shape and peak luminosity of the light curve are inconsistent with what would be expected for TDEs, further ruling out that possibility.

We argue instead that the transient in W0948+0318 corresponds to an SLSN. The shape of the $V_{\mathrm{CSS}}$ light curve and its peak luminosity are consistent with observations of other SLSNe. Furthermore, the UV/optical multiwavelength SED is consistent with a blackbody with a temperature of $13,600 \pm 500 \mathrm{~K}$ coupled with $\mathrm{H} \alpha$ and $\mathrm{H} \beta$ emission lines with large EWs of $960 \pm 340 \AA$ and $240 \pm 90 \AA$. These values are consistent with what has been previously observed for SLSNe. Using the conservative assumption that the temperature of the SED did not change throughout the transient, we estimate a total radiated energy of $E=(1.6 \pm 0.3) \times 10^{52} \mathrm{erg}$. As the temperature of the SLSN would have likely been much hotter closer to the peak luminosity, the total radiated energy might be higher. We further speculate that, because the dust temperature does not evolve throughout the transient or between the AllWISE and NEOWISE-R epochs, the location of the SLSN might have been within the torus of the AGN, as in that case the accretion disk emission would maintain the dust temperature regardless of the transient luminosity. Furthermore, the torus may be able to provide the dense CSM that is thought to be needed to make SNe superluminous.

Finally, we identify nine other highly variable, radioundetected WISE AGN candidates that could be analogs of W0948+0318 based on their mid-IR light curves. However, despite many of these objects being classified as type 1 AGNs, their $V_{\mathrm{CSS}}$ light curves do not show significant variability. This is further consistent with our suggestion that the transient of W0948+0318 corresponds to an SLSN in the torus of the AGN, as the majority of events could be obscured by dust in the torus. Based on the selection function of W0948+0318, we estimate that the rate of such transients is $>2 \times 10^{-7} \mathrm{yr}^{-1}$ per AGN. If these nine objects are true analogs to the transient in $\mathrm{W} 0948+0318$, we instead estimate a larger rate of $>7.5 \times 10^{-6} \mathrm{yr}^{-1}$ per AGN. If we consider these events in isolation and estimate the comoving volume in which they would have been selected by our study, we find that their rate would be too high to be compatible with being SLSNe unrelated to the AGN activity, further suggesting a relation between the two. However, as we cannot ascertain that these additional objects are true analogs to the transient in W0948 +0318 , our rate estimates are not stringent enough to confirm this scenario. Future studies relying on the much more extensive NEOWISE-R observations to identify new candidates will be needed to further constrain the nature of similar mid-IR transient events.

The authors would like to thank Krisztina Gabanyi and the anonymous referee for comments and suggestions that helped 
improve the article. The authors would also like to thank Christopher S. Kochanek for discussions and suggestions that helped make this a better paper. We would like to thank Hannah Earnshaw, Marianne Heida, Ashish Mahabal, and Gaël Noirot for obtaining observations used in this article. R. J. A. was supported by FONDECYT grant no. 1151408. Support for J.L.P. is provided in part by the Ministry of Economy, Development, and Tourisms Millennium Science Initiative through grant IC120009, awarded to The Millennium Institute of Astrophysics, MAS. H.D.J. is supported by the Basic Science Research Program through the National Research Foundation of Korea (NRF) funded by the Ministry of Education (NRF-2017R1A6A3A04005158). This research has made use of the NASA/IPAC Infrared Science Archive, which is operated by the Jet Propulsion Laboratory, California Institute of Technology, under contract with the National Aeronautics and Space Administration. This publication makes use of data products from the Wide-field Infrared Survey Explorer, which is a joint project of the University of California, Los Angeles, and the Jet Propulsion Laboratory/ California Institute of Technology, and NEOWISE, which is a project of the Jet Propulsion Laboratory/California Institute of Technology. WISE and NEOWISE are funded by the National Aeronautics and Space Administration. The Pan-STARRS1 Surveys (PS1) have been made possible through contributions of the Institute for Astronomy, the University of Hawaii, the Pan-STARRS Project Office, the Max-Planck Society and its participating institutes, the Max Planck Institute for Astronomy, Heidelberg, and the Max Planck Institute for Extraterrestrial Physics, Garching, Johns Hopkins University, Durham University, the University of Edinburgh, Queen's University Belfast, the Harvard-Smithsonian Center for Astrophysics, the Las Cumbres Observatory Global Telescope Network Incorporated, the National Central University of Taiwan, the Space Telescope Science Institute, the National Aeronautics and Space Administration under grant no. NNX08AR22G issued through the Planetary Science Division of the NASA Science Mission Directorate, the National Science Foundation under grant no. AST-1238877, the University of Maryland, and Eotvos Lorand University (ELTE), and the Los Alamos National Laboratory. Funding for SDSS-III has been provided by the Alfred P. Sloan Foundation, the Participating Institutions, the National Science Foundation, and the U.S. Department of Energy Office of Science. The SDSS-III website is http://www.sdss3. org/. SDSS-III is managed by the Astrophysical Research Consortium for the Participating Institutions of the SDSS-III Collaboration, including the University of Arizona, the Brazilian Participation Group, Brookhaven National Laboratory, Carnegie Mellon University, University of Florida, the French Participation Group, the German Participation Group, Harvard University, the Instituto de Astrofisica de Canarias, the Michigan State/Notre Dame/JINA Participation Group, Johns Hopkins University, Lawrence Berkeley National Laboratory, Max Planck Institute for Astrophysics, Max Planck Institute for Extraterrestrial Physics, New Mexico State University, New York University, Ohio State University, Pennsylvania State
University, University of Portsmouth, Princeton University, the Spanish Participation Group, University of Tokyo, University of Utah, Vanderbilt University, University of Virginia, University of Washington, and Yale University. The CSS survey is funded by the National Aeronautics and Space Administration under grant no. NNG05GF22G issued through the Science Mission Directorate Near-Earth Objects Observations Program. The CRTS survey is supported by the U.S. National Science Foundation under grant AST-0909182.

\section{Appendix A Highly Variable Radio-loud WISE AGNs}

As discussed in the main text, it is likely that the majority of AGN candidates with highly variable WISE fluxes that have a FIRST survey counterpart are blazars. Blazars can be bright throughout the electromagnetic spectrum, from the $\gamma$-rays to the radio. Here we look at the WISE colors of these sources to see whether they differ significantly from those that are not detected by FIRST.

Massaro et al. (2012), following the results of an earlier work, Massaro et al. (2011), developed a scheme to identify in the WISE data the counterparts of $\gamma$-ray sources using mid-IR colors. For this, Massaro et al. (2012) defined a WISE $\gamma$-ray strip (WGS) of WISE colors on which the counterparts of $\gamma$-ray sources are typically found. Specifically, Massaro et al. (2012) defined the boundaries of the WGS in the W1-W2 versus W2-W3, W2-W3 versus W3-W4, and W1-W2 versus W3W4 color-color diagrams, independently for BL Lacs and flatspectrum radio quasars. Massaro et al. (2012) furthermore define the total strip parameters $S_{b}$ and $S_{q}$ that quantify how consistent a given WISE source is with being in the WGS region given its colors and errors on those colors. A value of zero means that the source is inconsistent with the respective WGS.

Our group of highly variable WISE AGNs can be used to provide an interesting test on the WGS. While the WGS was defined specifically to aid in the identification of counterparts to $\gamma$-ray sources, we can test whether the WGS can be used to identify blazars simply from the WISE photometry. Specifically, we can test whether our highly variable WISE sources with radio counterparts, likely to be blazars, have different $S_{b}$ and $S_{q}$ parameters from those that do not have radio counterparts, and hence are unlikely to be blazars. Figure 12 shows the distribution of the $S_{b}$ and $S_{q}$ parameters for both subsamples. The objects with no radio detections are clearly more clustered toward $S_{b}=0$ and $S_{q}=0$. However, both samples have a large fraction of objects extending to similarly large $S_{b}$ and $S_{q}$ values, implying that these parameters are not a good discriminant of whether a WISE-selected AGN is a blazar or not. This is not surprising, as, in addition to the WGS not being built with this application in mind, the WGS projection in the W1-W2 versus W2-W3 plane overlaps strongly with the AGN selection criteria of Mateos et al. (2012), which does not aim to identify blazars, i.e., the WGS is effective at identifying all quasars, not just BL Lacs and flat-spectrum radio quasars. 


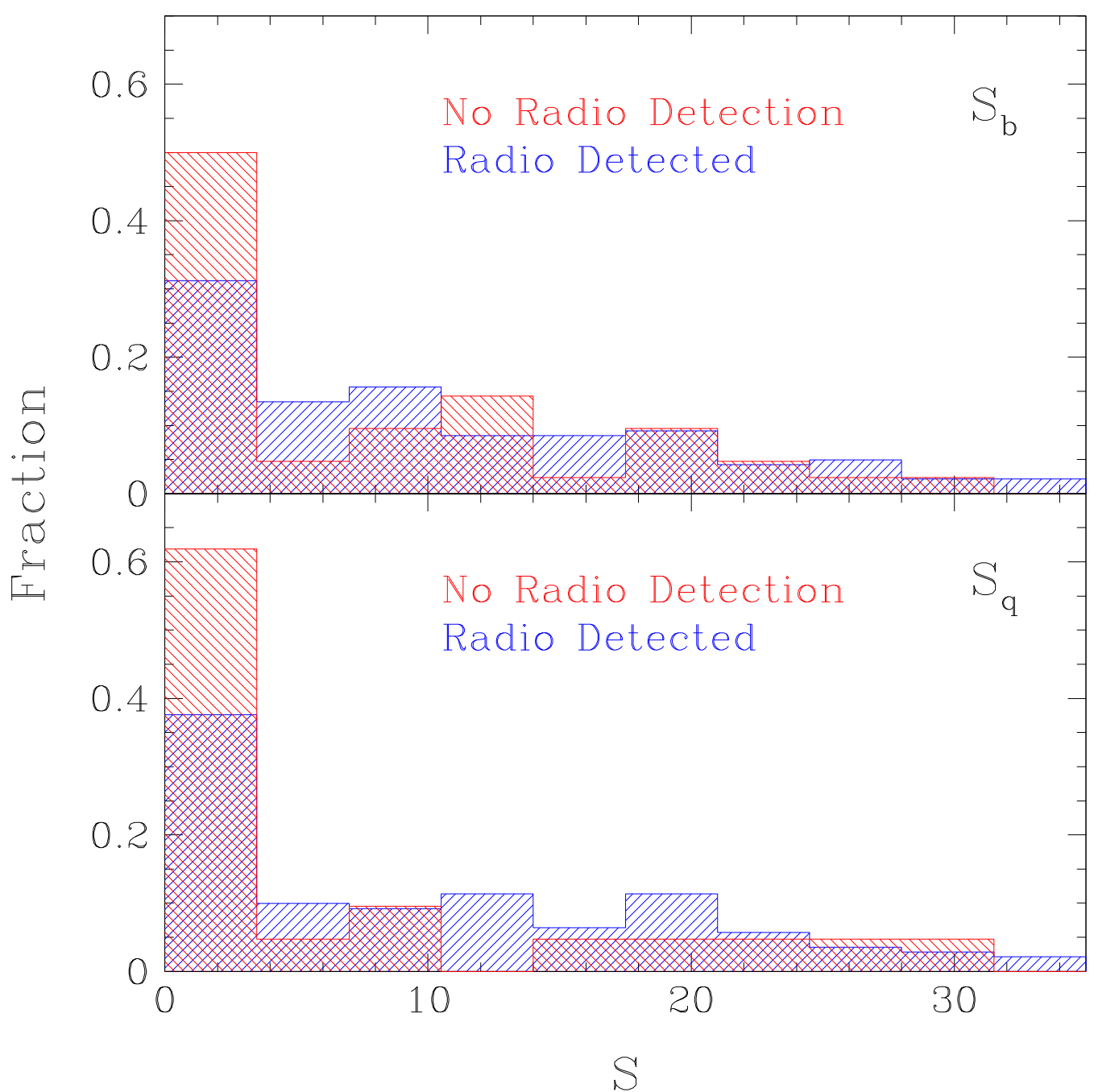

Figure 12. Distribution of the BL Lac $S_{b}$ (top) and flat-spectrum radio quasar $S_{q}$ (bottom) parameters of Massaro et al. (2012) for the highly variable WISE AGN candidates. The blue histograms show these values for the objects that are detected in the radio by FIRST, while the red histograms show them for the radio-undetected highly variable WISE AGN candidates. Objects with $S_{b}=0$ or $S_{q}=0$ are inconsistent with the respective WGS. The figure shows that the WGS is effective at identifying all quasars, not just BL Lacs or flat-spectrum radio quasars. 


\section{Appendix B \\ Optical and Mid-IR Light Curves for the Rest of the Sample}

Throughout the text the light curves of only 19 of the 45 highly variable, radio-undetected WISE sources have been presented. Here we provide, for completeness, the light curves of the remainder of the sample. These light curves are shown in Figure 13. Objects are arranged from left to right, top to bottom by their $V_{\mathrm{Css}}$-band magnitudes. As mentioned in the text, WISEA J144039.30+612748.1 is outside the footprint of the CRTS survey, and hence no optical light curve is available for it. We have put this object last.

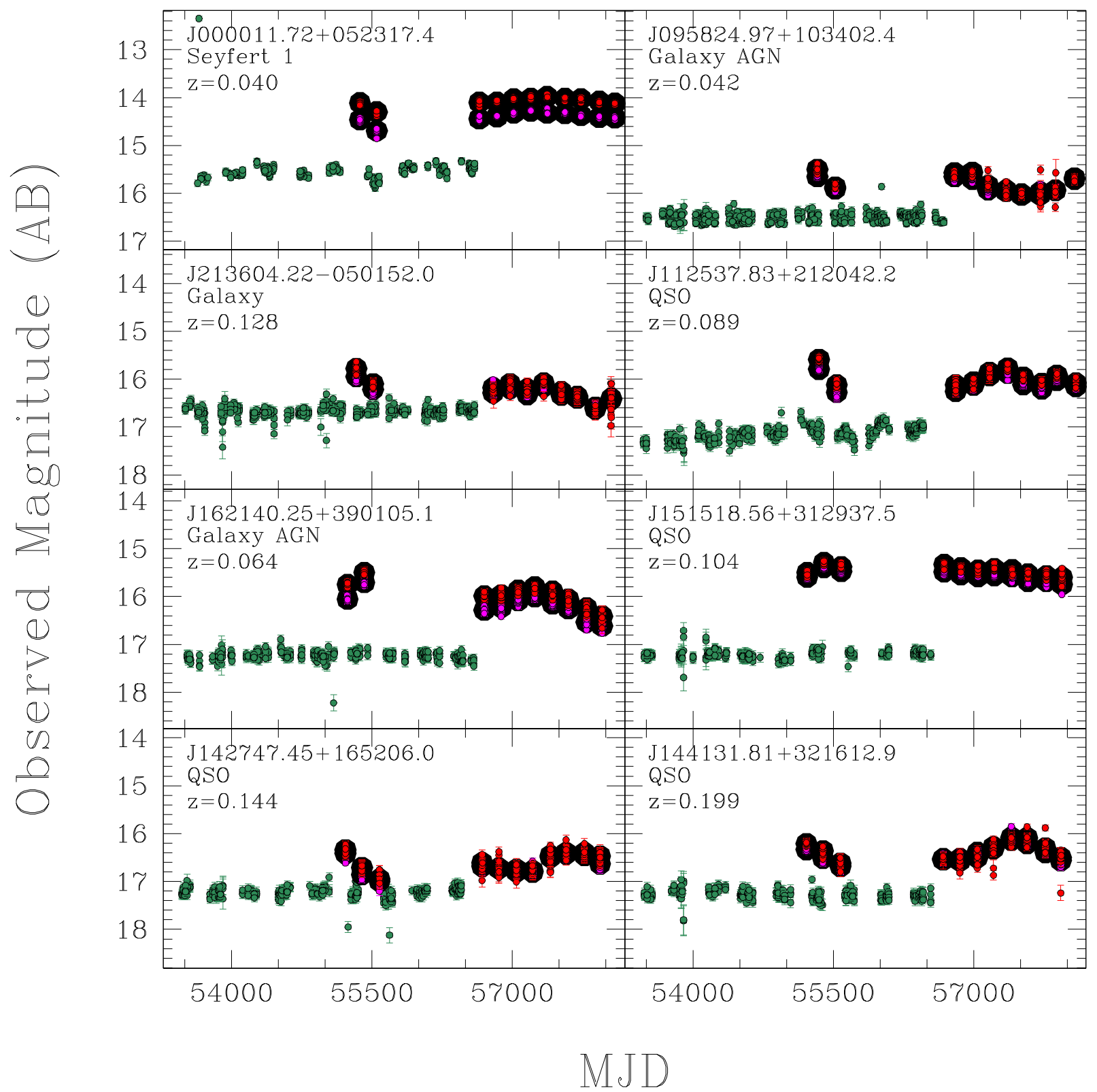

Figure 13. Light curves of the rest of the sample of highly variable WISE AGN candidates that are not detected by FIRST. Objects are arranged by their $V_{\mathrm{CSS}}$-band magnitude, and all magnitudes shown are in the AB system. Symbols and colors have the same meaning as in Figure 2. 


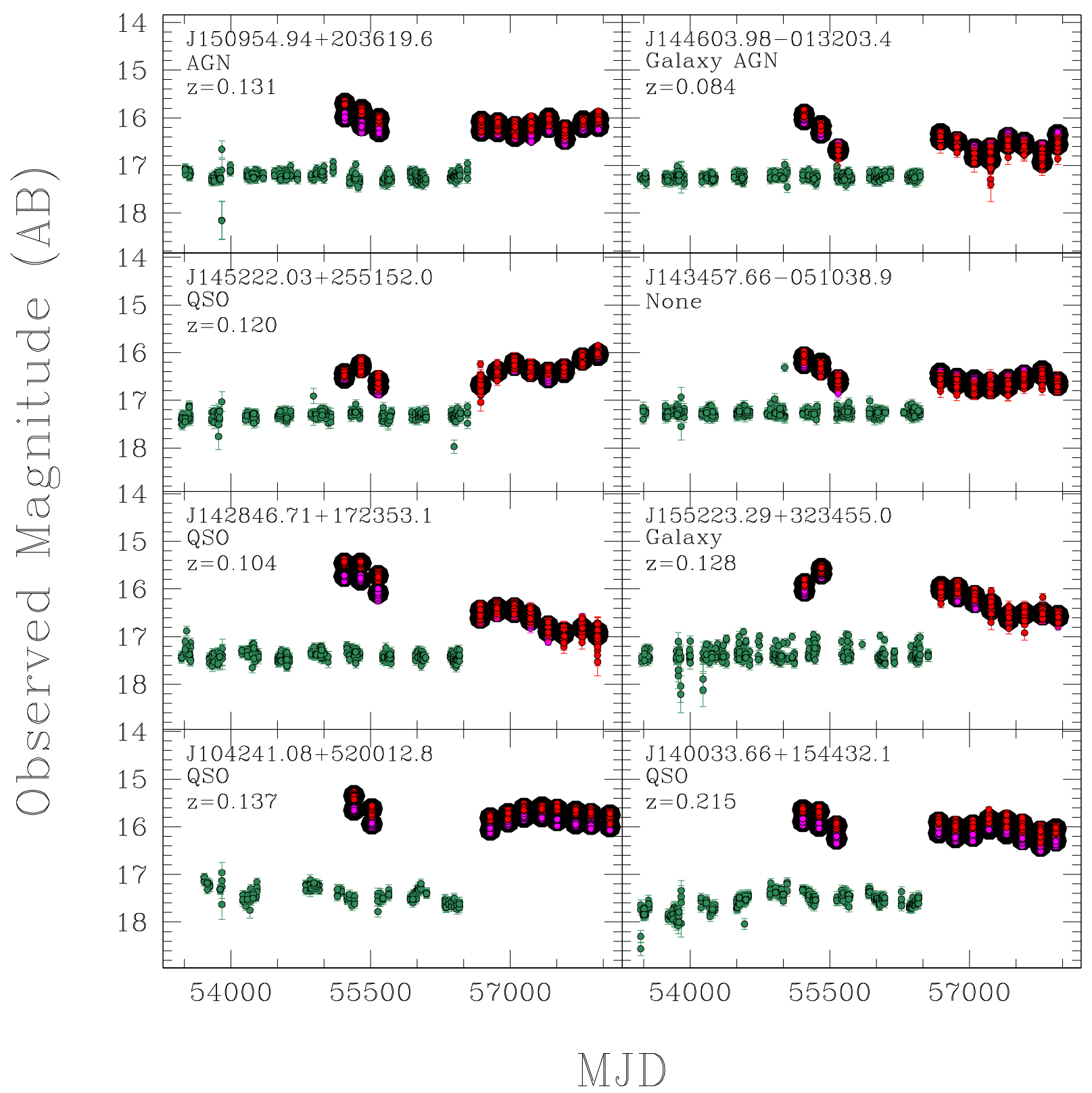

Figure 13. (Continued.) 


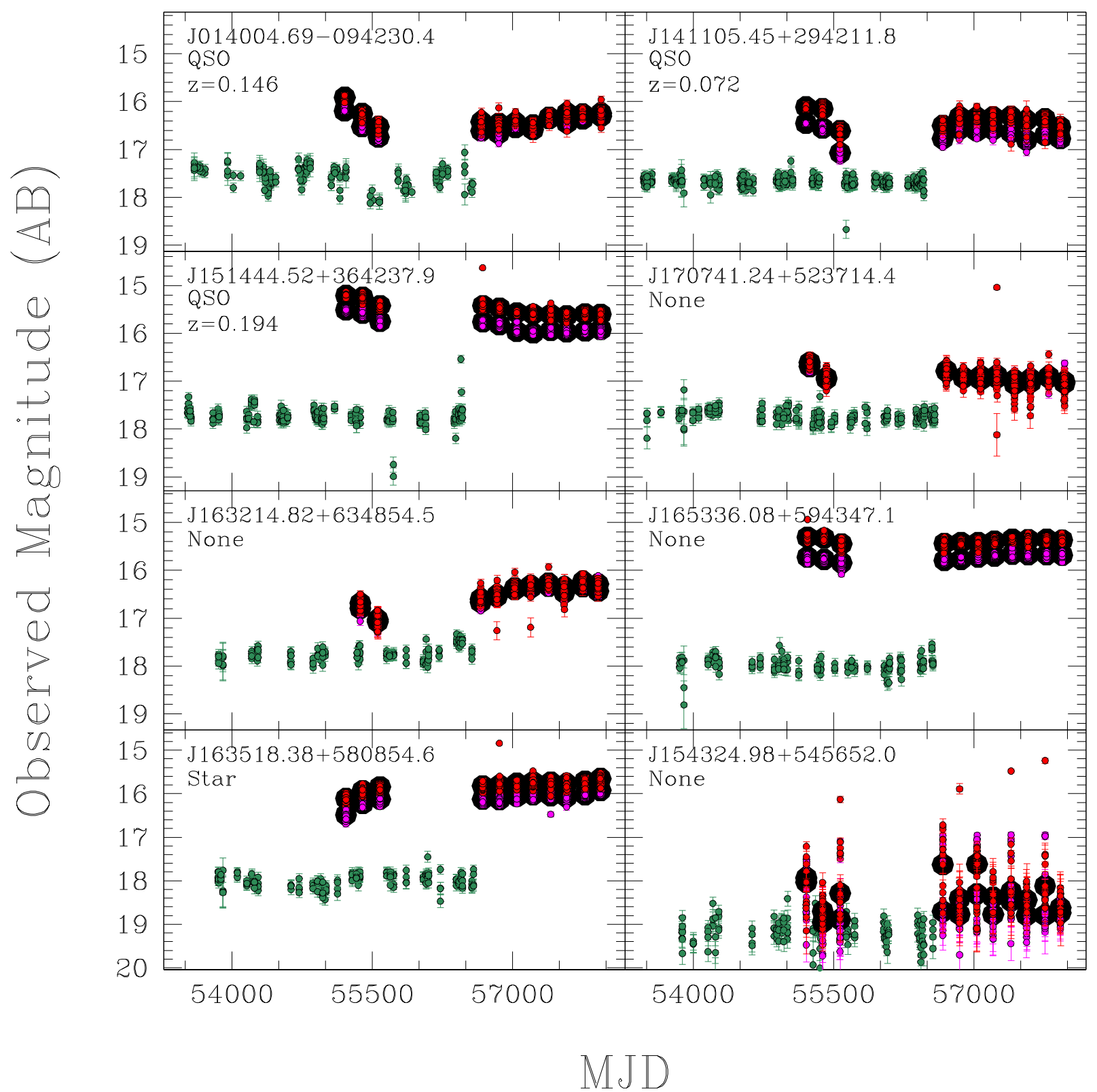

Figure 13. (Continued.) 


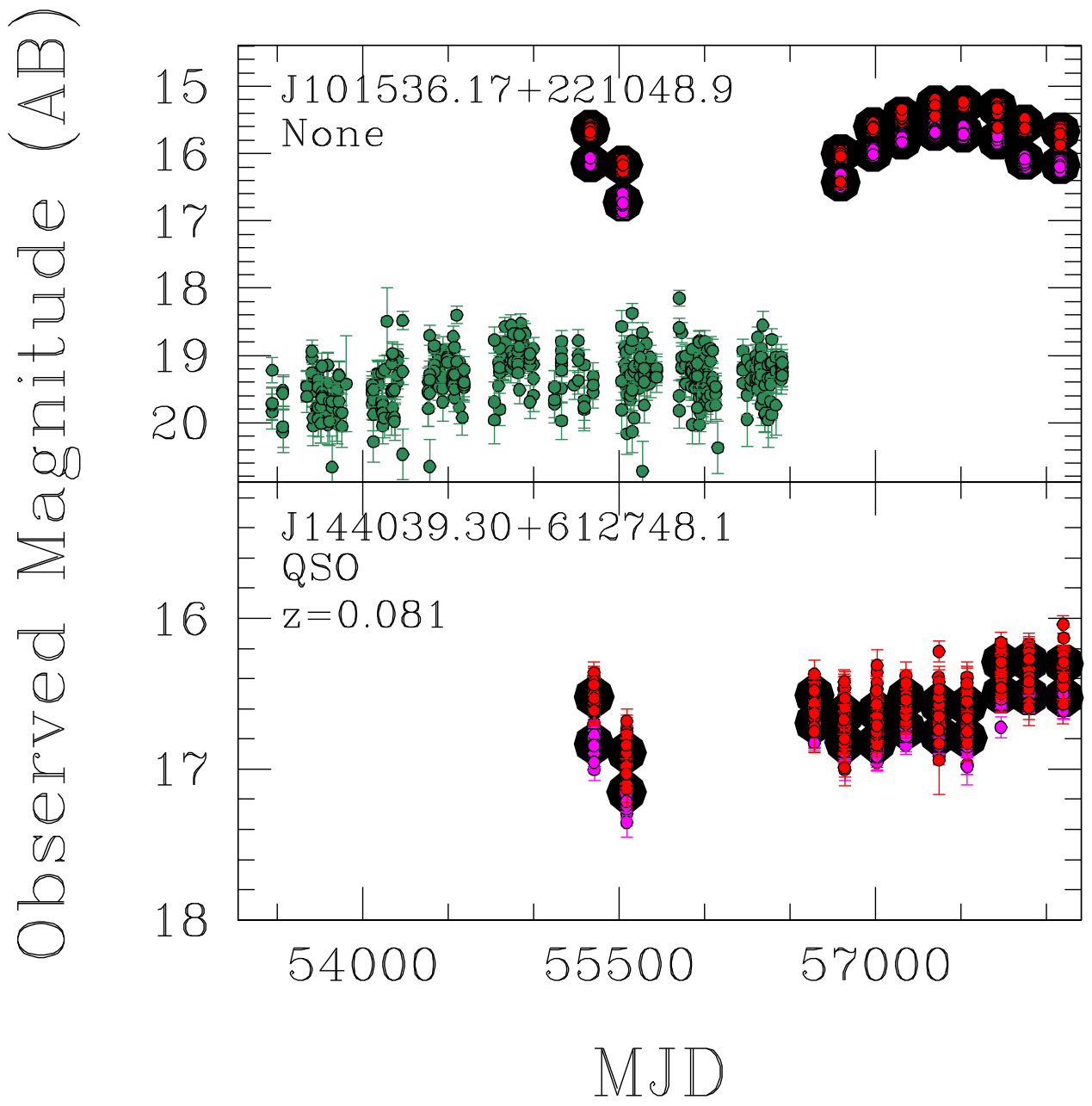

Figure 13. (Continued.) 


\section{Appendix C \\ Optical and Mid-IR Light Curves for the Rest of the Sample}

As discussed in Section 2.2, W0948+0318 was also identified by Graham et al. (2017) as one of 51 AGNs in the CRTS database whose light curves strongly deviated from a DRW model. Here we provide, for completeness, the optical and WISE light curves for 49 of the other 50 objects of that sample. The missing object, CRTS 2326+0005, is not found within the CRTS or WISE databases, suggesting a mistake in the coordinates provided by Graham et al. (2017). The CRTS and WISE light curves of these 49 objects are shown in Figure 14.

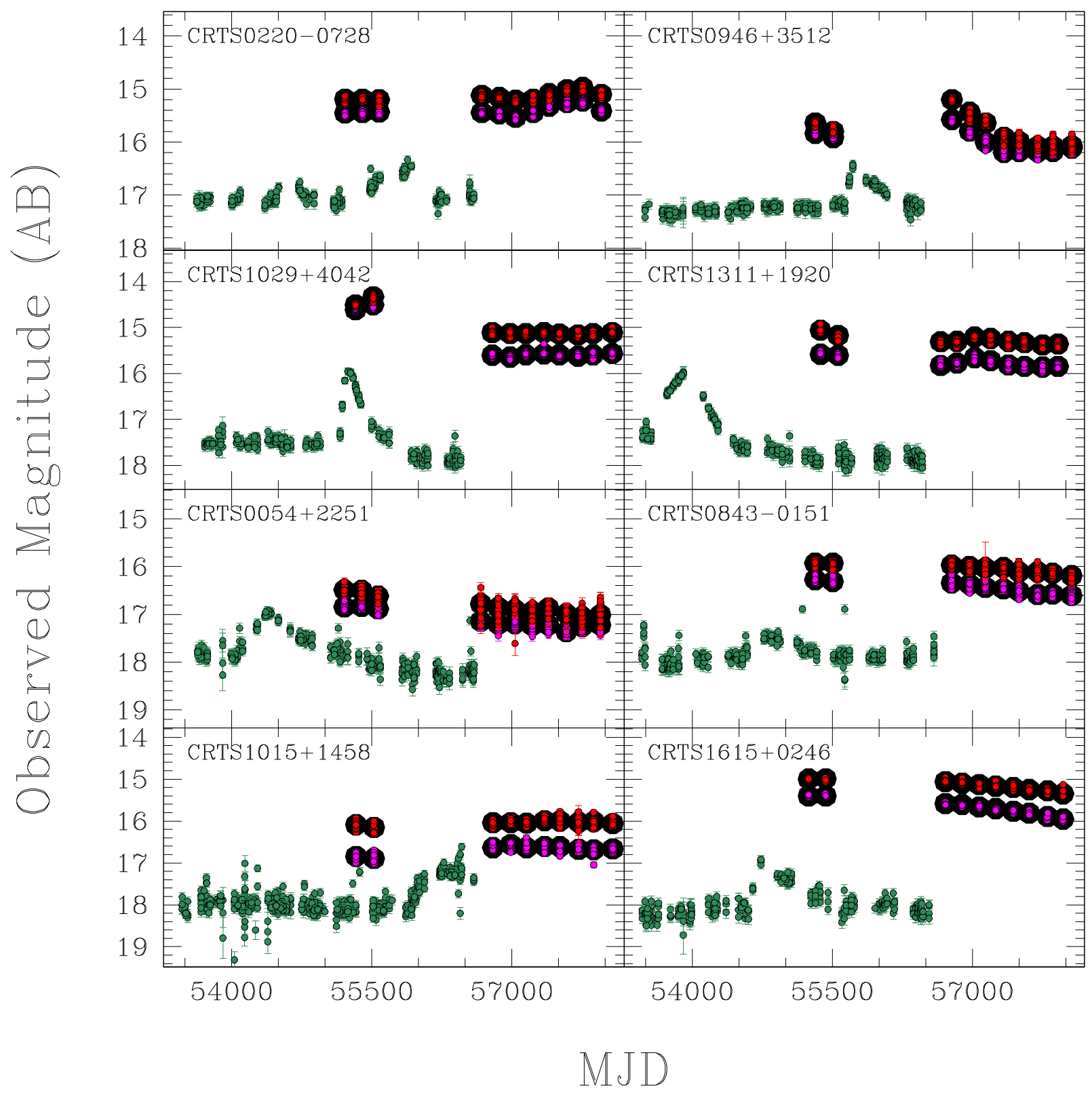

Figure 14. Light curves of the rest of the Graham et al. (2017) sample. Objects are arranged by their $V_{\mathrm{CSs}}$-band magnitude, and all magnitudes shown are in the $\mathrm{AB}$ system. Symbols and colors have the same meaning as in Figure 2. 


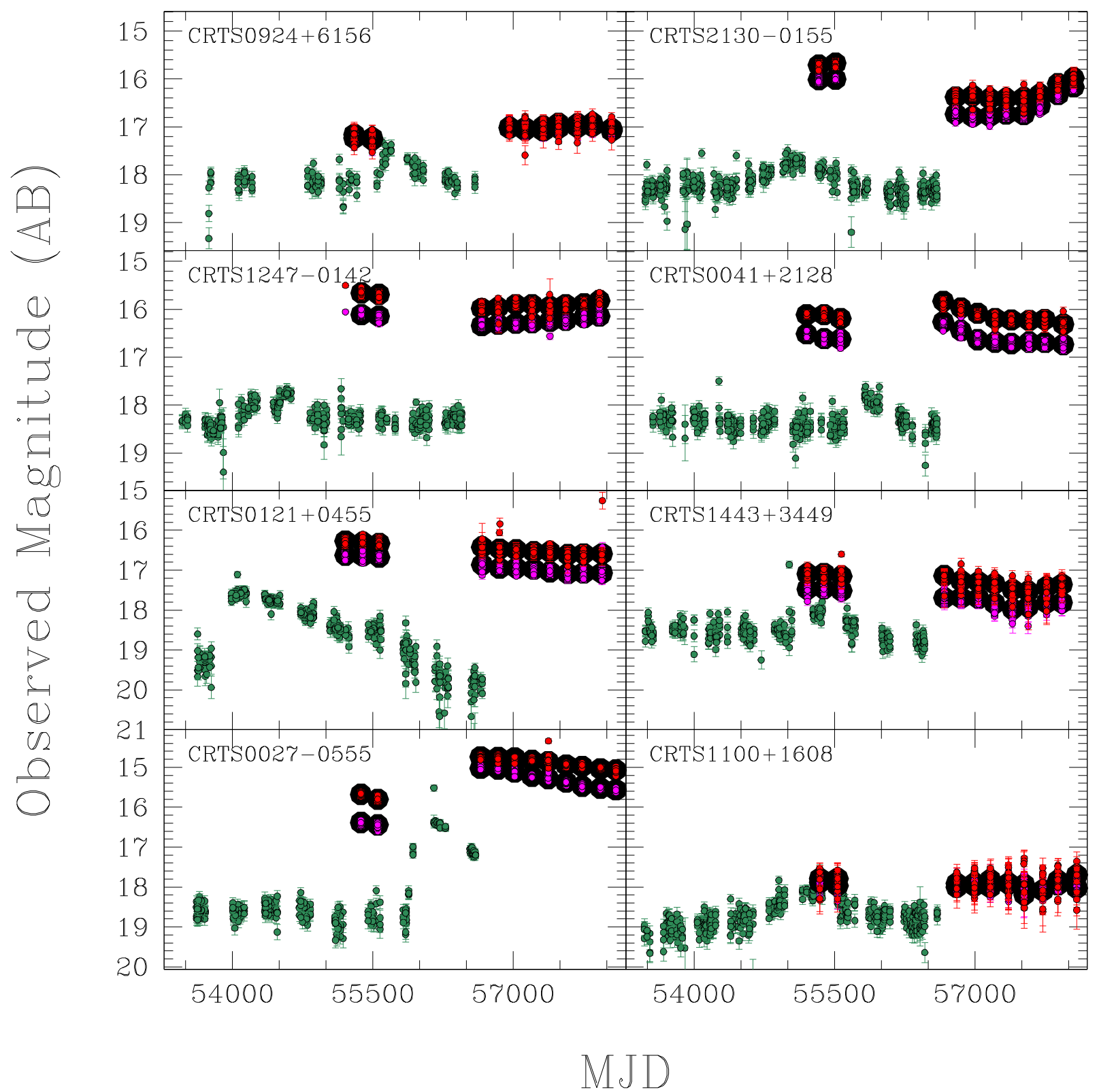

Figure 14. (Continued.) 


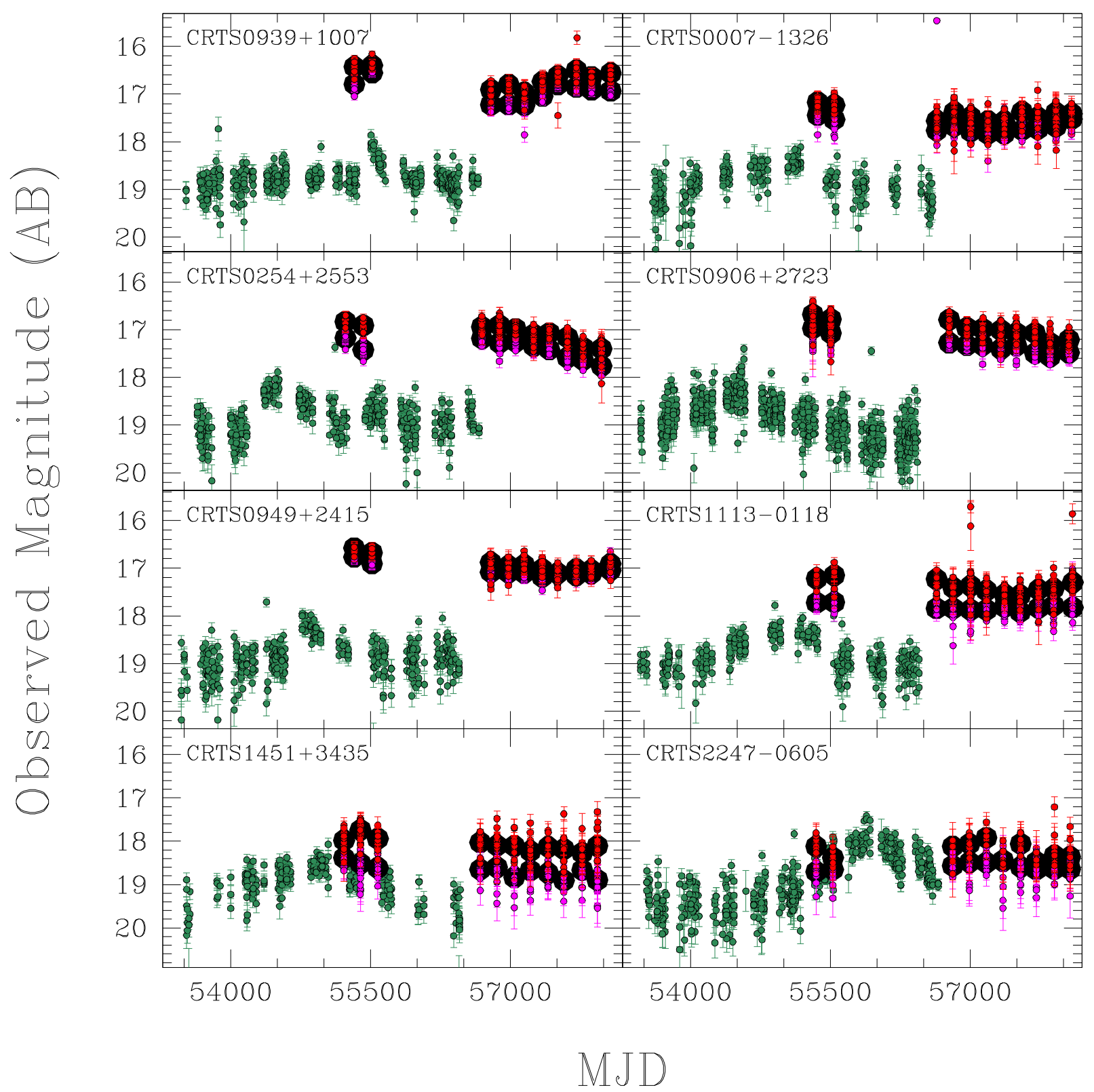

Figure 14. (Continued.) 


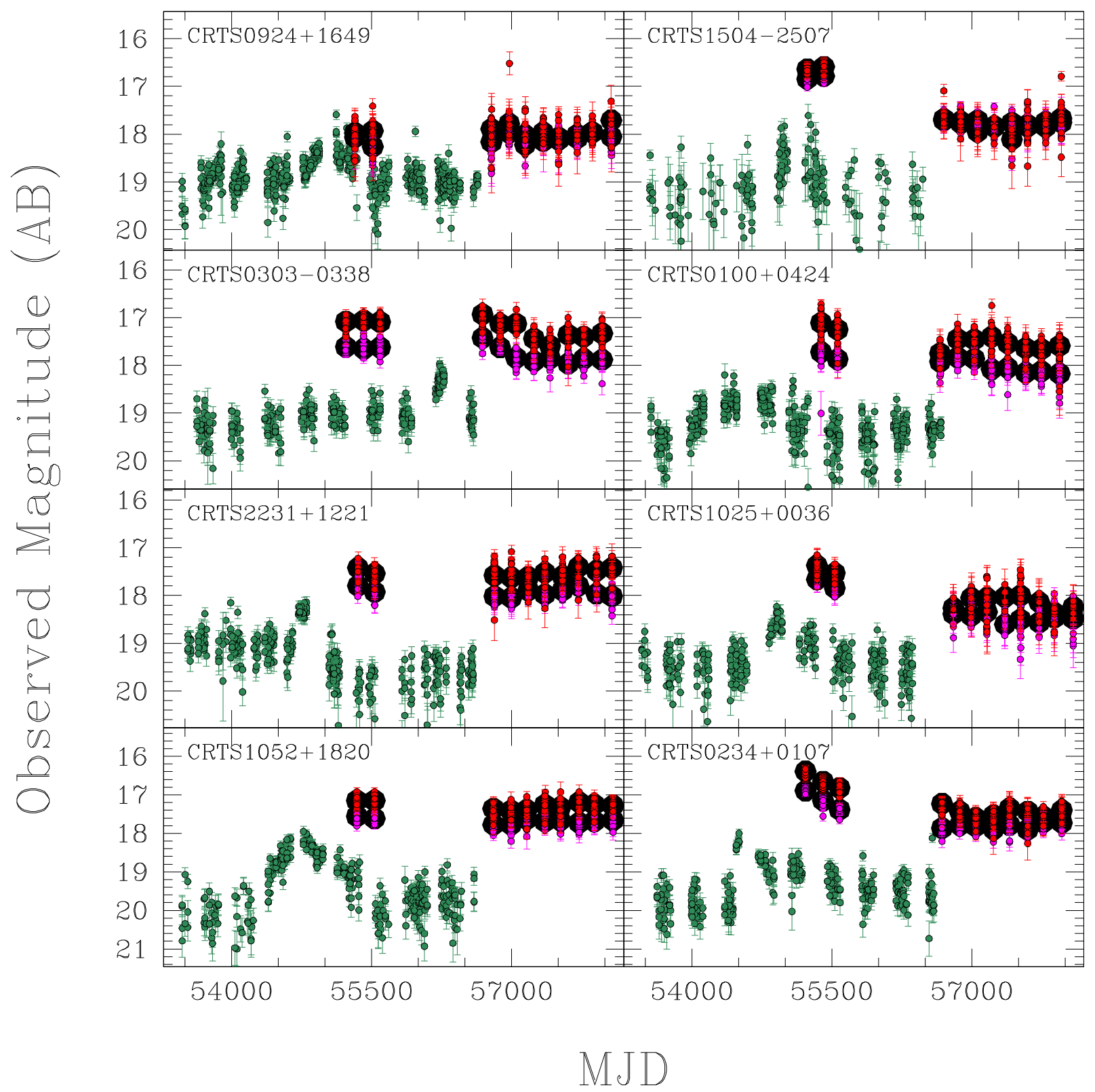

Figure 14. (Continued.) 


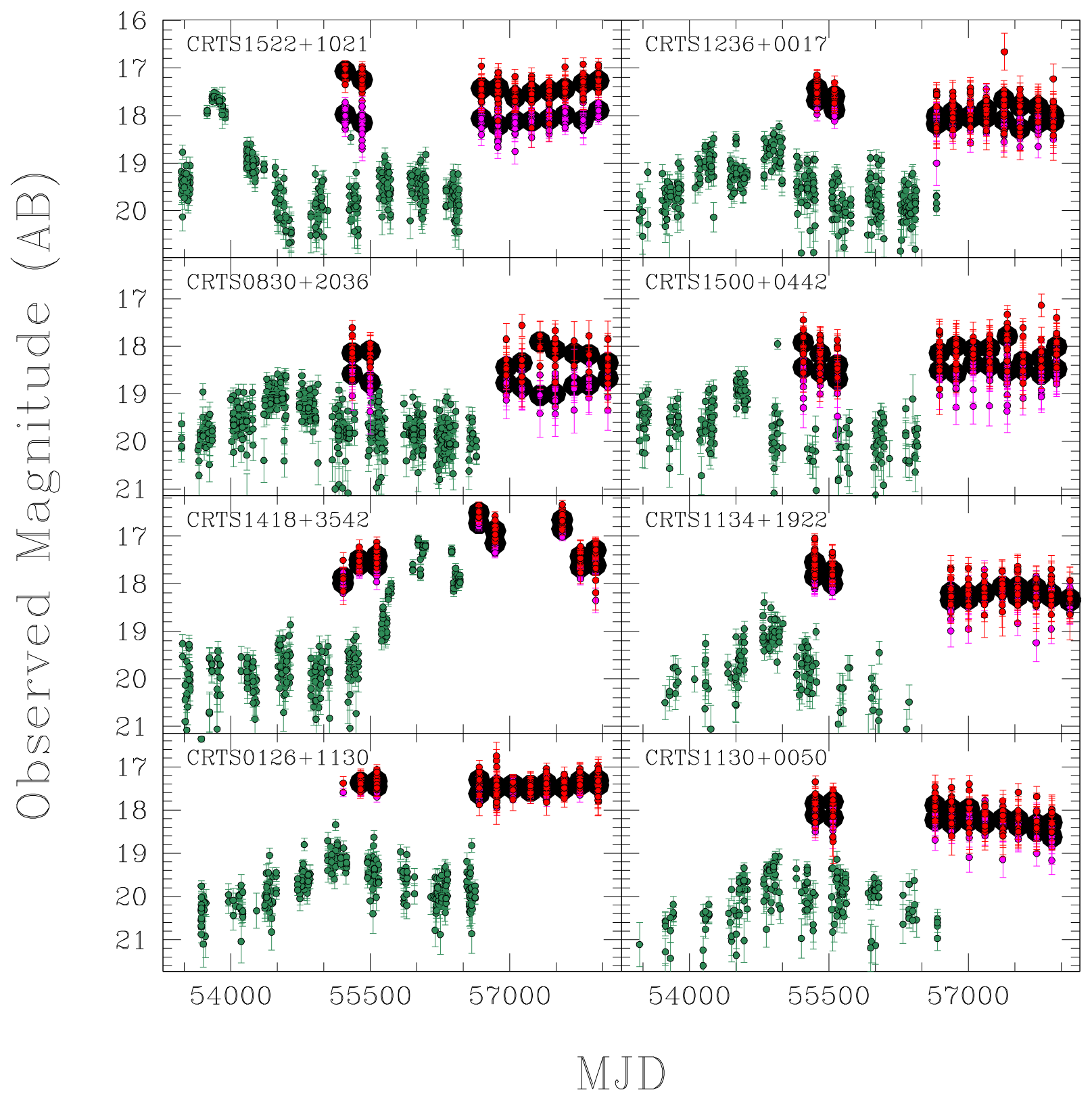

Figure 14. (Continued.) 


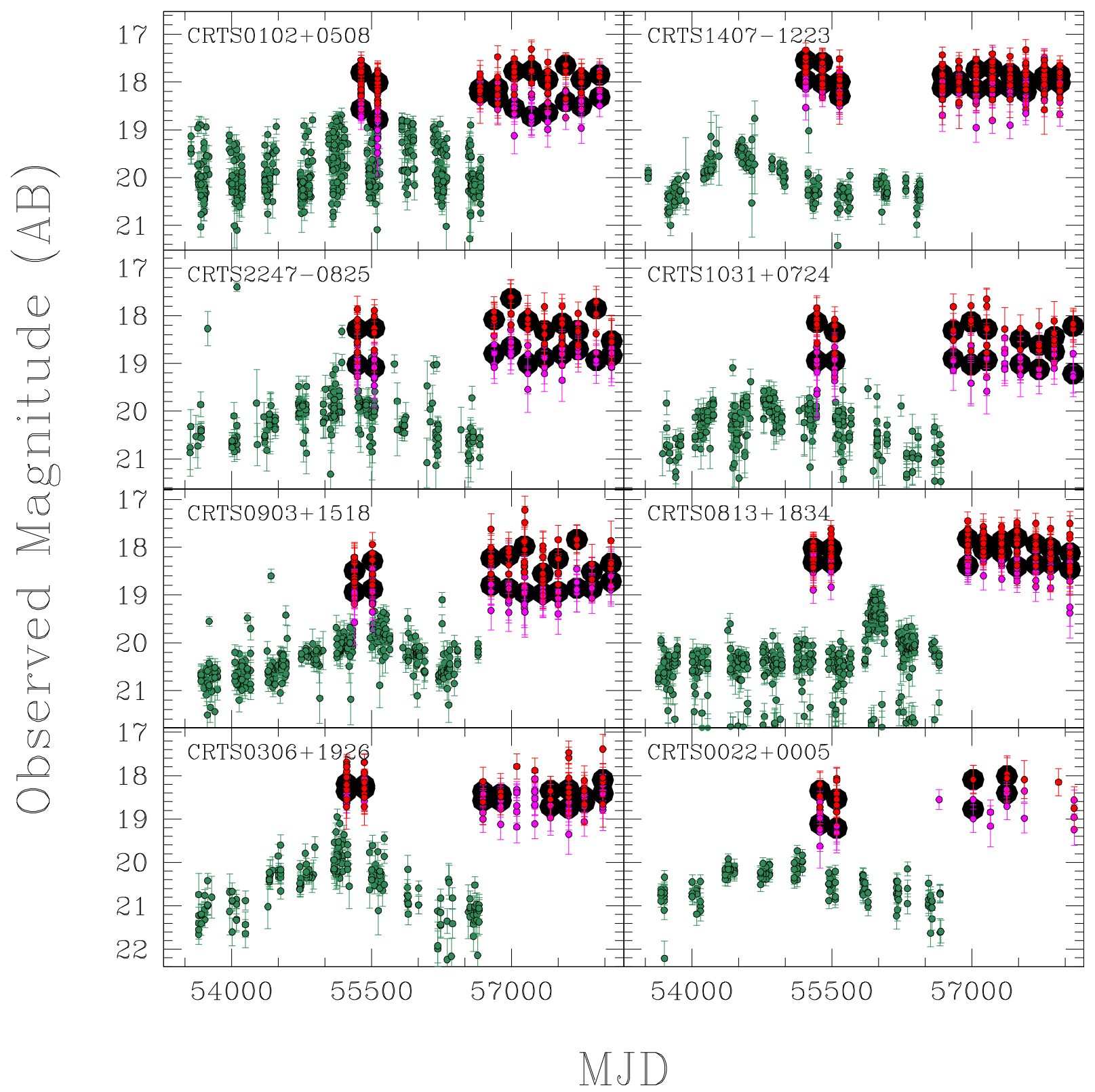

Figure 14. (Continued.) 


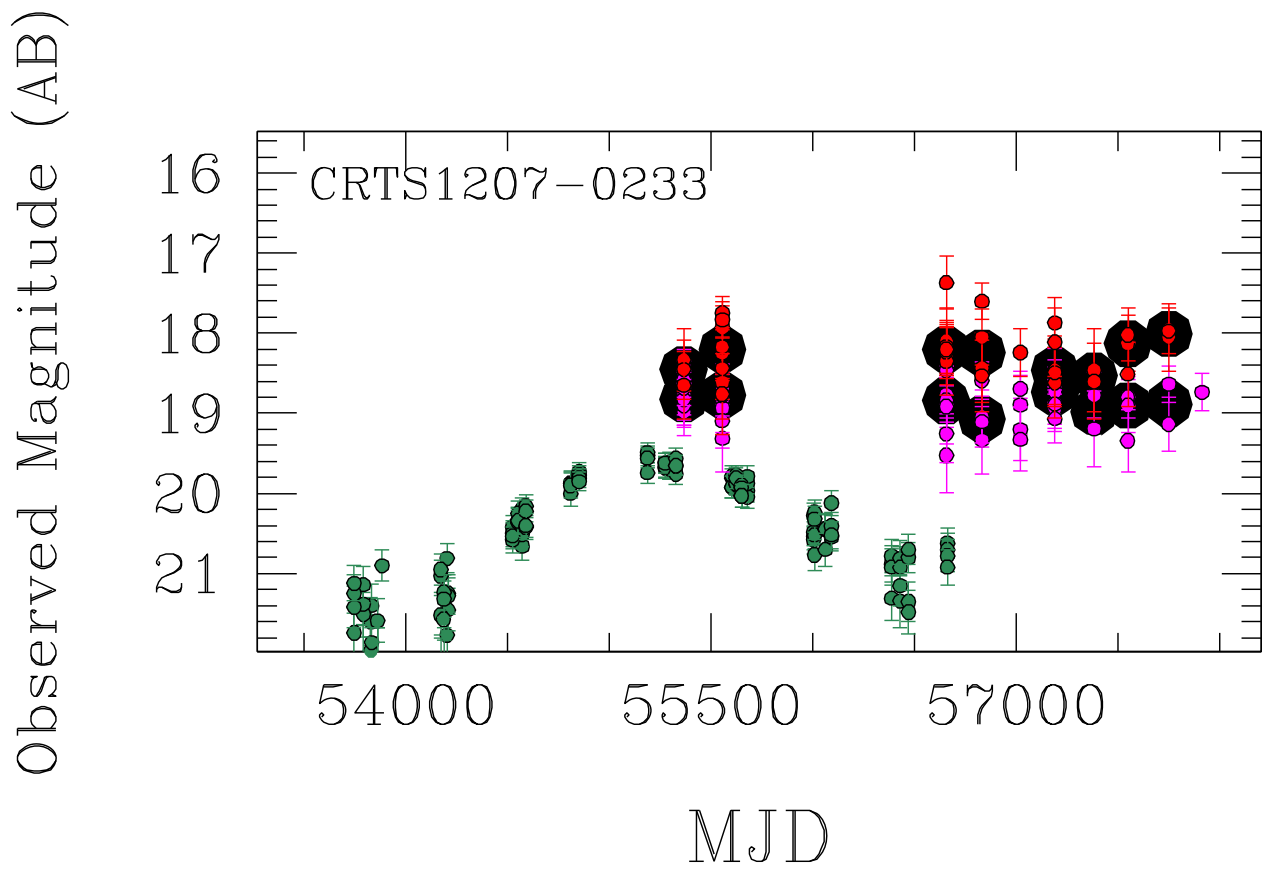

Figure 14. (Continued.)

\section{ORCID iDs}

R. J. Assef $\odot$ https://orcid.org/0000-0002-9508-3667

J. L. Prieto (1) https://orcid.org/0000-0003-0943-0026

D. Stern (10) https://orcid.org/0000-0003-2686-9241

R. M. Cutri (1) https://orcid.org/0000-0002-0077-2305

H. A. Flewelling (i) https://orcid.org/0000-0002-1050-4056

N. Kaiser (i) https://orcid.org/0000-0001-6511-4306

C. Waters (1) https://orcid.org/0000-0003-1989-4879

\section{References}

Albareti, F. D., Allende Prieto, C., Almeida, A., et al. 2017, ApJS, 233, 25

Ashby, M. L. N., Stern, D., Brodwin, M., et al. 2009, ApJ, 701, 428

Assef, R. J., Denney, K. D., Kochanek, C. S., et al. 2011, ApJ, 742, 93

Assef, R. J., Kochanek, C. S., Brodwin, M., et al. 2010, ApJ, 713, 970

Assef, R. J., Stern, D., Kochanek, C. S., et al. 2013, ApJ, 772, 26

Assef, R. J., Stern, D., Noirot, G., et al. 2018, ApJS, 234, 23

Becker, R. H., White, R. L., \& Helfand, D. J. 1995, ApJ, 450, 559

Bentz, M. C., Walsh, J. L., Barth, A. J., et al. 2009, ApJ, 705, 199

Blanchard, P. K., Nicholl, M., Berger, E., et al. 2017, ApJ, 843, 106

Brown, J. S., Kochanek, C. S., Holoien, T. W.-S., et al. 2018, MNRAS, 473, 1130

Caplar, N., Lilly, S. J., \& Trakhtenbrot, B. 2017, ApJ, 834, 111

Chambers, K. C., Magnier, E. A., Metcalfe, N., et al. 2016, arXiv:1612.05560

Collin, S., Kawaguchi, T., Peterson, B. M., \& Vestergaard, M. 2006, A\&A, 456, 75

Denney, K. D. 2012, ApJ, 759, 44

Denney, K. D., Peterson, B. M., Pogge, R. W., et al. 2009, ApJL, 704, L80

Dong, S., Shappee, B. J., Prieto, J. L., et al. 2016, Sci, 351, 257

Drake, A. J., Catelan, M., Djorgovski, S. G., et al. 2013, ApJ, 763, 32

Drake, A. J., Djorgovski, S. G., Mahabal, A., et al. 2009, ApJ, 696, 870

Drake, A. J., Djorgovski, S. G., Mahabal, A., et al. 2011, ApJ, 735, 106

Edelson, R., Gelbord, J. M., Horne, K., et al. 2015, ApJ, 806, 129

Flewelling, H. A., Magnier, E. A., Chambers, K. C., et al. 2016, arXiv:1612. 05243

Gallagher, S. C., Richards, G. T., Lacy, M., et al. 2007, ApJ, 661, 30

Gal-Yam, A. 2012, Sci, 337, 927

Gehrels, N. 1986, ApJ, 303, 336

Gezari, S., Chornock, R., Rest, A., et al. 2012, Natur, 485, 217
Godoy-Rivera, D., Stanek, K. Z., Kochanek, C. S., et al. 2017, MNRAS, 466,1428

Goodrich, R. W. 1989, ApJ, 342, 224

Graham, M. J., Djorgovski, S. G., Drake, A. J., et al. 2017, MNRAS, 470, 4112 Grupe, D. 2004, AJ, 127, 1799

Holoien, T. W.-S., Kochanek, C. S., Prieto, J. L., et al. 2016a, MNRAS, 455, 2918

Holoien, T. W.-S., Kochanek, C. S., Prieto, J. L., et al. 2016b, MNRAS, 463,3813

Holoien, T. W.-S., Prieto, J. L., Bersier, D., et al. 2014, MNRAS, 445, 3263

Jencson, J. E., Prieto, J. L., Kochanek, C. S., et al. 2016, MNRAS, 456, 2622

Kankare, E., Kotak, R., Mattila, S., et al. 2017, NatAs, 1, 865

Kasen, D., \& Bildsten, L. 2010, ApJ, 717, 245

Kelly, B. C., Bechtold, J., \& Siemiginowska, A. 2009, ApJ, 698, 895

Kozłowski, S. 2017, A\&A, 597, A128

Kozłowski, S., Kochanek, C. S., Ashby, M. L. N., et al. 2016, ApJ, 817, 119

Kozłowski, S., Kochanek, C. S., Stern, D., et al. 2010a, ApJ, 716, 530

Kozłowski, S., Kochanek, C. S., Stern, D., et al. 2010b, ApJ, 722, 1624

Kozłowski, S., Kochanek, C. S., Udalski, A., et al. 2010c, ApJ, 708, 927

Lawrence, A., Watson, M. G., Pounds, K. A., \& Elvis, M. 1987, Natur, 325,694

Leloudas, G., Fraser, M., Stone, N. C., et al. 2016, NatAs, 1, 0002

MacLeod, C. L., Ivezić, Ž, Kochanek, C. S., et al. 2010, ApJ, 721, 1014

Magnier, E. A., Chambers, K. C., Flewelling, H. A., et al. 2016, arXiv:1612. 05240

Mainzer, A., Bauer, J., Cutri, R. M., et al. 2014, ApJ, 792, 30

Mainzer, A., Bauer, J., Grav, T., et al. 2011, ApJ, 731, 53

Marconi, A., Risaliti, G., Gilli, R., et al. 2004, MNRAS, 351, 169

Martin, D. C., Fanson, J., Schiminovich, D., et al. 2005, ApJL, 619, L1

Martini, P., \& Weinberg, D. H. 2001, ApJ, 547, 12

Massaro, F., D'Abrusco, R., Ajello, M., Grindlay, J. E., \& Smith, H. A. 2011, ApJL, 740, L48

Massaro, F., D'Abrusco, R., Tosti, G., et al. 2012, ApJ, 750, 138

Mateos, S., Alonso-Herrero, A., Carrera, F. J., et al. 2012, MNRAS, 426, 3271

Mattila, S., Pérez-Torres, M., Efstathiou, A., et al. 2018, Sci, 361, 482

McHardy, I. M., Koerding, E., Knigge, C., Uttley, P., \& Fender, R. P. 2006, Natur, 444, 730

Morgan, C. W., Kochanek, C. S., Morgan, N. D., \& Falco, E. E. 2010, ApJ, 712,1129

Moriya, T. J., Tanaka, M., Morokuma, T., \& Ohsuga, K. 2017, ApJL, 843, L19 Nenkova, M., Sirocky, M. M., Nikutta, R., Ivezić, Ž, \& Elitzur, M. 2008, ApJ, 685,160

Osterbrock, D. E., \& Pogge, R. W. 1985, ApJ, 297, 166

Oyaizu, H., Lima, M., Cunha, C. E., et al. 2008, ApJ, 674, 768 
Padovani, P., Alexander, D. M., Assef, R. J., et al. 2017, A\&ARv, 25, 2 Pancoast, A., Brewer, B. J., \& Treu, T. 2011, ApJ, 730, 139

Peterson, B. M., Ferrarese, L., Gilbert, K. M., et al. 2004, ApJ, 613, 682

Quimby, R. M., Yuan, F., Akerlof, C., \& Wheeler, J. C. 2013, MNRAS, 431, 912

Rest, A., Foley, R. J., Gezari, S., et al. 2011, ApJ, 729, 88

Rest, A., Scolnic, D., Foley, R. J., et al. 2014, ApJ, 795, 44

Rest, A., Stubbs, C., Becker, A. C., et al. 2005, ApJ, 634, 1103

Ross, N. P., Ford, K. E. S., Graham, M., et al. 2018, MNRAS, 480, 4468

Schlafly, E. F., \& Finkbeiner, D. P. 2011, ApJ, 737, 103

Shakura, N. I., \& Sunyaev, R. A. 1973, A\&A, 24, 337

Skrutskie, M. F., Cutri, R. M., Stiening, R., et al. 2006, AJ, 131, 1163

Smith, N. 2014, ARA\&A, 52, 487

Smith, N., Li, W., Foley, R. J., et al. 2007, ApJ, 666, 1116
Stern, D., Assef, R. J., Benford, D. J., et al. 2012, ApJ, 753, 30

Stern, D., McKernan, B., Graham, M. J., et al. 2018, ApJ, 864, 27

Sukhbold, T., \& Woosley, S. E. 2016, ApJL, 820, L38

Szabo, T., Pierpaoli, E., Dong, F., Pipino, A., \& Gunn, J. 2011, ApJ, 736, 21

Vanden Berk, D. E., Wilhite, B. C., Kron, R. G., et al. 2004, ApJ, 601, 692

Vestergaard, M., \& Peterson, B. M. 2006, ApJ, 641, 689

Wang, T., Yan, L., Dou, L., et al. 2018, MNRAS, 477, 2943

Waters, C. Z., Magnier, E. A., Price, P. A., et al. 2016, arXiv:1612.05245

Woosley, S. E. 2010, ApJL, 719, L204

Wright, E. L., Eisenhardt, P. R. M., Mainzer, A. K., et al. 2010, AJ, 140, 1868 Yuan, H. B., Liu, X. W., \& Xiang, M. S. 2013, MNRAS, 430, 2188

Zu, Y., Kochanek, C. S., Kozłowski, S., \& Udalski, A. 2013, ApJ, 765, 106

Zu, Y., Kochanek, C. S., \& Peterson, B. M. 2011, ApJ, 735, 80 